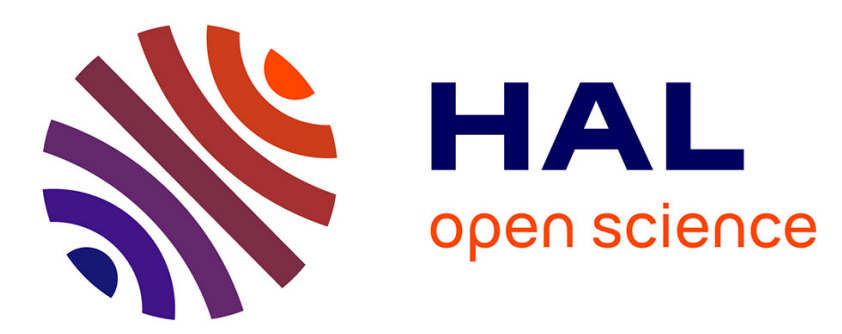

\title{
Stochastic modeling and identification of an uncertain computational dynamical model with random fields properties and model uncertainties
}

Anas Batou, Christian Soize

\section{> To cite this version:}

Anas Batou, Christian Soize. Stochastic modeling and identification of an uncertain computational dynamical model with random fields properties and model uncertainties. Archive of Applied Mechanics, 2013, 83 (6), pp.831-848. 10.1007/s00419-012-0720-7 . hal-00759467

\section{HAL Id: hal-00759467 \\ https://hal.science/hal-00759467}

Submitted on 30 Nov 2012

HAL is a multi-disciplinary open access archive for the deposit and dissemination of scientific research documents, whether they are published or not. The documents may come from teaching and research institutions in France or abroad, or from public or private research centers.
L'archive ouverte pluridisciplinaire HAL, est destinée au dépôt et à la diffusion de documents scientifiques de niveau recherche, publiés ou non, émanant des établissements d'enseignement et de recherche français ou étrangers, des laboratoires publics ou privés. 


\title{
Stochastic modeling and identification of an uncertain computational dynamical model with random fields properties and model uncertainties
}

Received: date / Accepted: date

\begin{abstract}
This paper is devoted to the construction and to the identification of a probabilistic model of random fields in presence of modeling errors, in high stochastic dimension and presented in the context of computational structural dynamics. Due to the high stochastic dimension of the random quantities which have to be identified using statistical inverse methods (challenging problem), a complete methodology is proposed and validated. The parametric-nonparametric (generalized) probabilistic approach of uncertainties is used to perform the prior stochastic models: (1) system-parameters uncertainties induced by the variabilities of the material properties are described by random fields for which their statistical reductions are still in high stochastic dimension and (2) model uncertainties induced by the modeling errors are taken into account with the nonparametric probabilistic approach in high stochastic dimension. For these two sources of uncertainties, the methodology consists in introducing prior stochastic models described with a small number of parameters which are simultaneously identified using the maximum likelihood method and experimental responses. The steps of the methodology are explained and illustrated through an application.
\end{abstract}

Keywords High stochastic dimension - Structural dynamics · Random fields · Model uncertainties · Nonparametric probabilistic approach

\section{Introduction}

We are interested in the response of dynamical systems for which the parameters of the materials are variable and not perfectly known. This situation induces uncertainties in the material properties, which are mainly due to the manufacturing process of materials and structural elements, due to the assembly of the structure and finally, due to the life cycle of this structure. The estimation of the responses in the very low-frequency range (the first two or three resonances) is generally robust with respect to uncertainties in the material properties (but is sensitive to other sources of uncertainties such as uncertainties in the boundary conditions). Nevertheless, the sensitivity of the dynamical response with respect to materials uncertainties becomes non negligible when the frequency increases in the low-frequency range and in particular, in the medium-frequency range which contains numerous elastic modes which are very sensitive to uncertainties. Nevertheless, this type of uncertainties, which

\footnotetext{
A. Batou

Université Paris-Est, Laboratoire Modélisation et Simulation Multi Echelle, MSME UMR 8208 CNRS, 5 bd Descartes, 77454 Marne-la-Vallee, France

Tel.: +33-160957792

Fax: +33-160957799

E-mail: anas.batou@univ-paris-est.fr

C. Soize

Université Paris-Est, Laboratoire Modélisation et Simulation Multi Echelle, MSME UMR 8208 CNRS, 5 bd Descartes, 77454 Marne-la-Vallee, France
} 
corresponds to system-parameters uncertainties, cannot explain all the differences between experimental measurements and the predictions performed with the computational models. The modeling errors appear during the construction of the nominal computational model (mathematical-mechanical modeling) and depend on the accuracy which is chosen for constructing the computational model. Modeling errors induce model uncertainties which have to be taken into account in order to obtain robust predictions.

Concerning system-parameters uncertainties, the main method is based on the use of the parametric probabilistic approach which allows the uncertain system parameters of the nominal model to be taken into account through the introduction of prior probability models of uncertain parameters (see for instance $[10 ; 20 ; 25 ; 26 ; 32]$ ). Such an approach consists (1) either in directly constructing the probability distributions of the random quantities using the available information and the Maximum Entropy Principle (MaxEnt) introduced by Jaynes [16] in the context of Information Theory developed by Shannon [28] (see for instance [18; 17]), (2) or in constructing an adapted representation based on polynomial chaos extensions of the random quantities (see for instance $[10 ; 19]$ and for an application to random fields of material properties $[4 ; 6 ; 36])$. It should be noted that the methodologies which are useful to construct polynomial chaos expansions of random fields have been introduced in [10;34] and have also been analyzed in $[30 ; 39 ; 38]$ for arbitrary measures. The polynomial chaos expansions of random fields, and application to stochastic boundary value problems, have generated many works in the last decade (see for instance $[5 ; 8 ; 9 ; 11 ; 13 ; 21 ; 23 ; 26 ; 40]$ ).

There are two main methods to take into account model uncertainties induced by modeling errors. (1) The first one consists in introducing a probabilistic model of the output-prediction error which is the difference between the real system output and the model output. A posterior probabilistic model can be constructed using, for instance, the Bayesian approach (see for instance [2]) but in such a case, experimental data are absolutely required. (2) The second one is based on the nonparametric probabilistic approach of modeling errors which has been introduced in [29] (see Appendix A) as another possible way to the use of the output-prediction-error method in order to take into account modeling errors. The nonparametric probabilistic approach consists in directly constructing the stochastic modeling of the operators of the nominal computational model and can be used even if no available data are available.

The objective of this work is to propose a methodology for a statistical inverse problem, in high stochastic dimension, related to uncertain computational models in structural dynamics. Due to the high stochastic dimension of the random quantities which have to be identified through a computational model, this challenging problem is very difficult to solve. The novelty of this work is to propose a complete methodology which is adapted to the high stochastic dimension for computational models in structural dynamics and which consists in constructing prior stochastic models described with a small number of parameters and then, to identify these parameters using the maximum likelihood method and experimental responses of the dynamical system.

At the knowledge of the authors, this is the first work which is devoted to such a case consisting in constructing and in identifying the stochastic model of random fields in presence of modeling errors taken into account with the nonparametric probabilistic approach, for the high stochastic dimension case. The two sources of uncertainties are the system-parameters uncertainties and the model uncertainties induced by modeling errors. The generalized probabilistic approach of uncertainties has recently been proposed in order to take into account these two sources of uncertainties. In the application presented in [32], the uncertain system parameters are homogeneous material properties modeled by random variables (therefore the stochastic dimension of the probabilistic model of the uncertain system-parameters is very low). In this paper, the first objective is to construct a prior probabilistic model of uncertainties using the generalized probabilistic approach and for which the uncertain system-parameters are material properties fields modeled by random fields for which their statistical reductions stay in high stochastic dimension. The second objective is to propose a global uncertainty quantification in identifying the parameters of the probabilistic model constructed using the parametric-nonparametric probabilistic approach, called the generalized probabilistic approach. Unlike the method adopted in [32], the parameters relative to system-parameters uncertainties and parameters relative to model uncertainties are identified in a single step using all the information given by the experimental responses. The last objective is to analyze the role played by mass modeling errors in the medium-frequency range through the development of an application. Concerning the field of the framework related to computational structural dynamics, the methodology proposed is adapted 
to the low- and medium-frequency ranges. The medium-frequency dynamical analysis is of primary importance in many industrial application fields such as the vibration analysis of launch vehicles, the vibroacoustic analysis of automotive vehicles, etc. In this paper, the different steps and the feasibility of the methodology proposed are illustrated through an application but the methodology is general and can be applied to more complex structures.

In Section 2, the objectives and main steps of the methodology we propose in this paper are summarized. Section 3 is devoted to the presentation of the reduced nominal computational model. In Section 4 , the stochastic computational model is constructed using the reduced nominal computational model and a generalized probabilistic approach of uncertainties. Section 5 deals with the identification of the parameters of the stochastic computational model. Finally, in Section 6, we apply the methodology to a computational model related to a beam structure for which the bulk modulus and the shear modulus are random fields.

\section{Objectives and methodology.}

In this paper, we are interested in a computational model in structural dynamics which requires stochastic models in high stochastic dimension for

- uncertain system parameters (such as a random field for which its statistical reduction stays in high stochastic dimension).

- model uncertainties induced by modeling errors (such as ten thousands independent random variable for instance).

These two sources of uncertainties have to be taken into account in order to predict the dynamical responses of the computational model with a good robustness. We then propose an adapted methodology for identifying the stochastic models of uncertainties which is relevant of statistical inverse problems in high stochastic dimension. It should be noted that uncertainties relative to the external loads are not taken into account. If existed, the stochastic modeling of such uncertain external loads could be carried out using the usual parametric approach and could be added without difficulties. The methodology proposed can be summarized in four steps.

- Step 1: Construction of a prior probabilistic model of uncertainties depending on a small number of unknown parameters which have to be identified. Consequently, the prior stochastic model must be carefully constructed using all the information available for the problem. Due to the small number of parameters which control the stochastic models of uncertainties and which have to be identified, the statistical inverse problem can effectively be solved even in the high stochastic dimension context.

-Step 2: Due to the presence of high stochastic dimension random quantities inducing high computational cost and due to the use of the nonparametric probabilistic approach of model uncertainties, it is necessary to construct a reduced-order computational model deduced from the computational model. -Step 3: Choice and construction of a stochastic solver. There are two classes of stochastic solvers. The first class of techniques rely on spectral stochastic methods such as the polynomial chaos expansion (see $[10 ; 19]$ ) or alternative methods such as the optimal separated representations (see [22]) and generalized spectral decomposition method (see [24]). Taking into account the high stochastic dimension case of the problem under consideration, these techniques cannot easily be used at the present time and still require additional researches. Consequently, we propose to use the second class of techniques based on sampling method such as the Monte Carlo method whose convergence is independent of the stochastic dimension.

-Step 4: Solving the statistical inverse problem which is then equivalent to identify the optimal values of the parameters of the prior probabilistic model of uncertainties constructed in Step 1, using the stochastic reduced-order model constructed in Step 2, the maximum likelihood method and the Monte Carlo method as stochastic solver.

If a large amount of experimental data was available, a posterior probabilistic model of uncertainties could be constructed using, for instance, the Bayesian approach. We do not consider such a case in the present paper.

The four steps are detailed in Sections 4 and 5. 


\section{Reduced nominal computational model}

\subsection{Nominal computational model}

We are interested in the dynamical response of a three-dimensional damped structure having a linear behavior and subjected to external forces.

The structure is made up of a linear dissipative elastic medium (viscoelastic medium with instantaneous memory) occupying an open bounded domain $\Omega$ of $\mathbb{R}^{3}$, with boundary $\partial \Omega=\Gamma_{0} \cup \Gamma$, in a Cartesian frame $\left(O x_{1} x_{2} x_{3}\right)$. Let $\mathbf{x}=\left(x_{1}, x_{2}, x_{3}\right)$ be any point in $\Omega$. The external unit normal to $\partial \Omega$ is denoted by $\mathbf{n}=\left(n_{1}, n_{2}, n_{3}\right)$. The boundary value problem is formulated in the frequency domain with the Fourier transform convention $\mathbf{g}(\omega)=\int_{\mathbb{R}} e^{-i \omega t} \mathbf{g}(t) d t$ in which $i=\sqrt{-1}$ and where $\omega$ is the real frequency (in $\mathrm{rad} / \mathrm{s})$. Let $\mathbf{u}(\mathbf{x}, \omega)=\left(u_{1}(\mathbf{x}, \omega), u_{2}(\mathbf{x}, \omega), u_{3}(\mathbf{x}, \omega)\right)$ be the displacement field defined on $\Omega$ with values in $\mathbb{C}^{3}$. On part $\Gamma_{0}$ of the boundary, there is a Dirichlet condition $\mathbf{u}=0$. Consequently, there will be no rigid body displacements. This hypothesis can be released in the developments presented in this paper. A surface force field, $\mathbf{f}^{\text {surf }}(\mathbf{x}, \omega)=\left(f_{1}^{\text {surf }}(\mathbf{x}, \omega), f_{2}^{\text {surf }}(\mathbf{x}, \omega), f_{3}^{\text {surf }}(\mathbf{x}, \omega)\right)$ with values in $\mathbb{C}^{3}$, is applied to part of $\Gamma$ on the boundary. In addition, there is a volume force field, $\mathbf{f}^{\mathrm{vol}}(\mathbf{x}, \omega)=\left(f_{1}^{\mathrm{vol}}(\mathbf{x}, \omega), f_{2}^{\mathrm{vol}}(\mathbf{x}, \omega), f_{3}^{\mathrm{vol}}(\mathbf{x}, \omega)\right)$ with values in $\mathbb{C}^{3}$, applied in $\Omega$. We are interested in the linear response around a static equilibrium considered as the reference configuration defined by $\Omega$. It is assumed that there is no prestress. The boundary value problem in the frequency domain is written, for all real $\omega$, as

$$
\begin{array}{cc}
-\omega^{2} \rho \mathbf{u}-\operatorname{div} \boldsymbol{\sigma}=\mathbf{f}^{\text {vol }} & \text { in } \Omega, \\
\mathbf{u}=\mathbf{0} & \text { on } \Gamma_{0}, \\
\boldsymbol{\sigma} \mathbf{n}=\mathbf{f} & \text { on } \Gamma,
\end{array}
$$

where $\rho(\mathbf{x})$ is the mass density which is assumed to be a positive-valued bounded function on $\Omega$, $\boldsymbol{\sigma}(\mathbf{x}, \omega)$ is the second-order stress tensor, in which $\{\operatorname{div} \boldsymbol{\sigma}(\mathbf{x}, \omega)\}_{j}=\sum_{k=1}^{3} \partial \sigma_{j k}(\mathbf{x}, \omega) / \partial x_{k}$ and where $\{\boldsymbol{\sigma}(\mathbf{x}, \omega) \mathbf{n}\}_{j}=\sum_{k=1}^{3} \sigma_{j k}(\mathbf{x}, \omega) n_{k}(\mathbf{x})$. Let $\boldsymbol{\varepsilon}(\mathbf{x}, \omega)$ be the strain tensor defined by

$$
\varepsilon_{j k}(\mathbf{x}, \omega)=\frac{1}{2}\left(\frac{\partial u_{j}(\mathbf{x}, \omega)}{\partial x_{k}}+\frac{\partial u_{k}(\mathbf{x}, \omega)}{\partial x_{j}}\right) .
$$

The stress tensor $\boldsymbol{\sigma}(\mathbf{x}, \omega)$ will be related to the strain tensor $\varepsilon(\mathbf{x}, \omega)$ by a constitutive equation which is written for a nonhomogeneous anisotropic dissipative elastic medium as

$$
\sigma_{h \ell}(\mathbf{x}, \omega)=a_{h \ell j k}(\mathbf{x}) \varepsilon_{j k}(\mathbf{x}, \omega)+i \omega b_{h \ell j k}(\mathbf{x}) \varepsilon_{j k}(\mathbf{x}, \omega),
$$

in which $a_{h \ell j k}(\mathbf{x})$ and $b_{h \ell j k}(\mathbf{x})$ are the fourth-order real tensors related to the elastic and dissipative parts and which must satisfy symmetry and positiveness properties. Some of the system parameters such as, for example, the mass density, the elastic and dissipative material properties represented by tensor-valued fields $a_{h \ell j k}$ and $b_{h \ell j k}$ can be uncertain. Let $\mathbf{x} \mapsto \mathbb{h}(\mathbf{x})$ denote the vector field with values in $\mathbb{R}^{n_{p}}$ whose components are the $n_{p}$ uncertain system parameters. Note that, the elasticity and dissipative tensor-valued fields which are represented by $2 \times 2$ real-valued fields for an isotropic dissipative material and by $2 \times 21$ real-valued fields for an anisotropic material, are rewritten as a vector-valued fields denoted by $\{\mathfrak{h}(\mathbf{x}), \mathbf{x} \in \Omega\}$.

The computational model is constructed using the finite element (FE) method applied to the weak formulation of the boundary value problem defined by Eqs. (1) to (3). Let $\mathbf{x}^{1}, \ldots, \mathbf{x}^{N}$ be the set of the $N$ integration points of the finite elements. The vector-field $h(\mathbf{x})$ is then discretized in the integration points. Let be $n_{d}=N \times n_{p}$. Let $\mathbf{h}=\left(\mathbb{h}\left(\mathbf{x}^{1}\right), \ldots, \mathbb{h}\left(\mathbf{x}^{N}\right)\right) \in \mathbb{R}^{n_{d}}$ be the vector of the discretized uncertain system parameters. The structure is analyzed on the frequency band of analysis $B=\left[0, \omega_{\max }\right]$. Therefore, for all $\omega \in B$, the vector $\mathbf{y}(\omega)$ of the $m$ degrees-of-freedom is the solution of the following matrix equation

$$
\left(-\omega^{2}[\mathbf{M}(\mathbf{h})]+i \omega[\mathbb{D}(\mathbf{h})]+[\mathbb{K}(\mathbf{h})]\right) \mathbf{y}(\omega)=\widetilde{\mathbf{f}}(\omega),
$$

in which $[\mathbb{M}(\mathbf{h})],[\mathbb{D}(\mathbf{h})]$ and $[\mathbb{K}(\mathbf{h})]$ are the $(m \times m)$ mass, damping and stiffness matrices and where $\widetilde{\mathbf{f}}(\omega)$ is the vector of the external forces. 
3.2 Reduced-order nominal computational model

It is assumed that there is no rigid body displacements (this hypothesis can be released without difficulties). The reduced nominal computation model is constructed using the modal analysis. Let $\mathcal{C}_{h}$ be the admissible set for the vector $\mathbf{h}$. Then for all $\mathbf{h}$ in $\mathcal{C}_{h}$, the $n$ first eigenvalues $0<\lambda_{1}(\mathbf{h}) \leq \lambda_{2}(\mathbf{h}) \leq$ $\ldots \leq \lambda_{n}(\mathbf{h})$ associated with the elastic modes $\left\{\phi_{1}(\mathbf{h}), \phi_{2}(\mathbf{h}), \ldots, \phi_{n}(\mathbf{h})\right\}$ are solutions of the following generalized eigenvalue problem

$$
[\mathbb{K}(\mathbf{h})] \phi(\mathbf{h})=\lambda(\mathbf{h})[\mathbb{M}(\mathbf{h})] \phi(\mathbf{h}) .
$$

The reduced-order nominal computation model is obtained by projecting the nominal computation model on the subspace spanned by the $n$ first elastic modes calculated using Eq. (5). Let $[\Phi(\mathbf{h})]$ be the $m \times n$ matrix whose columns are the $n$ first elastic modes. We then introduce the approximation $\mathbf{y}^{(n)}(\omega)$ of $\mathbf{y}(\omega)$ which is written as

$$
\mathbf{y}^{(n)}(\omega)=[\Phi(\mathbf{h})] \mathbf{q}(\omega)
$$

in which the vector $\mathbf{q}(\omega)$ is the vector of the $n$ generalized coordinates and is the solution of the following reduced matrix equation

$$
\left(-\omega^{2}[M(\mathbf{h})]+i \omega[D(\mathbf{h})]+[K(\mathbf{h})]\right) \mathbf{q}(\omega)=\mathbf{f}(\omega ; \mathbf{h}),
$$

in which $[M(\mathbf{h})]=[\Phi(\mathbf{h})]^{T}[\mathbb{M}(\mathbf{h})][\Phi(\mathbf{h})],[D(\mathbf{h})]=[\Phi(\mathbf{h})]^{T}[\mathbb{D}(\mathbf{h})][\Phi(\mathbf{h})]$ and $[K(\mathbf{h})]=[\Phi(\mathbf{h})]^{T}[\mathbb{K}(\mathbf{h})][\Phi(\mathbf{h})]$ are the $n \times n$ mass, damping and stiffness generalized matrices, and where $\mathbf{f}(\omega ; \mathbf{h})=[\Phi(\mathbf{h})]^{T} \tilde{\mathbf{f}}(\omega) \in \mathbb{R}^{n}$ is the vector of the generalized forces.

The dimension $n$ of the reduced-order computational model is fixed to the value for which a good convergence of $\mathbf{y}^{(n)}(\omega)$ is reached for all $\omega$ in frequency band $B$ and for all $\mathbf{h}$ in $\mathcal{C}_{h}$.

\section{Stochastic reduced-order computational model}

The stochastic reduced-order computational model is constructed using the generalized probabilistic approach of uncertainties introduced in [32]. This approach allows both system-parameters uncertainties and uncertainties due to modeling errors (model uncertainties) to be taken into account.

\subsection{Prior probabilistic model for system-parameters uncertainties}

The material property fields of the dynamical system are modeled by random fields which means that the vector-field $\{\mathfrak{h}(\mathbf{x}), \mathbf{x} \in \Omega\}$ is modeled by the random vector-field $\{\mathbb{H}(\mathbf{x}), \mathbf{x} \in \Omega\}$. There are different ways to construct the prior stochastic model of random field $H$ :

- (1) Using an algebraic stochastic representation of random field $\mathbb{H}$ which is written, for all $\mathbf{x}$ in $\Omega$, as $\mathbb{H}(\mathbf{x})=\mathbb{E}(\mathbb{G}(\mathbf{x}))$ in which $\mathbb{E}$ is a given nonlinear deterministic mapping and where $\{\mathbb{G}(\mathbf{x}), \mathbf{x} \in \Omega\}$ is a given random field for which the probability law (system of marginal probability distributions) is completely defined and for which a generator of independent realizations is available (see for instance $[31 ; 35]$ for tensor-valued random fields adapted to Elasticity Theory).

- (2) If the mean function and the covariance function of random field $\mathbb{H}$ are known (that is the case if an algebraic stochastic representation of $\mathbb{H}$ has been constructed or if experimental data are available for estimating these two functions with a sufficient accuracy), then, under certain hypotheses, a statistical reduction can be constructed using the Karhunen-Loeve expansion. Let $E\{$.$\} be the mathematical expectation and let \underline{H}(\mathbf{x})=E\{\mathbb{H}(\mathbf{x})\}$ be the mean function. The random field $\mathbb{H}(\mathbf{x})$ can then be written as $\mathbb{H}(\mathbf{x})=\underline{H}(\mathbf{x})+\sum_{j=1}^{\mu} \sqrt{\gamma_{j}} \eta_{j} \psi^{j}(\mathbf{x})$, in which $\gamma_{1}, \ldots, \gamma_{\mu}$ are the eigenvalues and $\psi^{1}(\mathbf{x}), \ldots, \psi^{\mu}(\mathbf{x})$ are the associated eigenvectors of the covariance operator defined by the covariance function $C_{\mathbb{H}}\left(\mathbf{x}, \mathbf{x}^{\prime}\right)=E\left\{(\mathbb{H}(\mathbf{x})-\underline{H}(\mathbf{x}))\left(\mathbb{H}\left(\mathbf{x}^{\prime}\right)-\underline{H}\left(\mathbf{x}^{\prime}\right)\right)^{T}\right\}$, where $\eta_{1}, \ldots, \eta_{\mu}$ are uncorrelated second-order centered random variables and where $\mu$ is the order of the statistical reduction whose value is determined by a convergence analysis. The random vector $\boldsymbol{\eta}=\left(\eta_{1}, \ldots, \eta_{\mu}\right)$ (which is not Gaussian) can be represented using a polynomial chaos expansion (see for instance $[4 ; 6 ; 10 ; 12 ; 19])$. 
In this paper, we use an algebraic stochastic representation as a prior stochastic model. The vectorvalued random field $\{H(\mathbf{x}), \mathbf{x} \in \Omega\}$ is then completely defined and is discretized at the same integration points as the vector-valued field $\{\mathfrak{h}(\mathbf{x}), \mathbf{x} \in \Omega\}$. We then introduce the random vector $\mathbf{H}=\left(\mathbb{H}\left(\mathbf{x}^{1}\right), \ldots, \mathbb{H}\left(\mathbf{x}^{N}\right)\right)$ with values in $\mathbb{R}^{n_{d}}$, defined on a probability space $(\Theta, \mathcal{T}, \mathcal{P})$ and for which the prior probability density function $p_{\mathbf{H}}(\mathbf{h})$ with respect to $d \mathbf{h}$ is known. For any realization $\mathbf{H}(\theta)$ of the random vector $\mathbf{H}$, with $\theta$ in $\Theta$, the realizations $\left[\mathbf{M}_{\mathrm{par}}(\theta)\right],\left[\mathbf{D}_{\mathrm{par}}(\theta)\right]$ and $\left[\mathbf{K}_{\mathrm{par}}(\theta)\right]$ of the $(n \times n)$ random generalized mass, damping and stiffness matrices $\left[\mathbf{M}_{\mathrm{par}}\right],\left[\mathbf{D}_{\mathrm{par}}\right]$ and $\left[\mathbf{K}_{\mathrm{par}}\right]$ are then defined (for $A$ denoting $M, D$ or $K$ ) by

$$
\left[\mathbf{A}_{\text {par }}(\theta)\right]=[A(\mathbf{H}(\theta))] .
$$

By construction, each matrix $\left[\mathbf{M}_{\mathrm{par}}(\theta)\right],\left[\mathbf{D}_{\mathrm{par}}(\theta)\right]$ or $\left[\mathbf{K}_{\mathrm{par}}(\theta)\right]$ is positive definite and therefore, the Cholesky decomposition yields (for $A$ denoting $M, D$ or $K$ ),

$$
\left[\mathbf{A}_{\mathrm{par}}(\theta)\right]=\left[\mathbf{L}_{A}(\theta)\right]^{T}\left[\mathbf{L}_{A}(\theta)\right] .
$$

The prior probability density function $p_{\mathbf{H}}(\mathbf{h})$ of the random vector $\mathbf{H}$ depends on parameters such as the mean values and the coefficients of variation of the components of $\mathbf{H}$. Let $\boldsymbol{\alpha}_{\text {par }}$ be the vector whose components are the parameters of the prior probability density function which is rewritten as $p_{\mathbf{H}}\left(\mathbf{h} ; \boldsymbol{\alpha}_{\text {par }}\right)$ and which has to be identified using experimental responses. As explained in Section 2, the size of vector $\boldsymbol{\alpha}_{\text {par }}$ is small by construction of the prior stochastic model even if the statistical reductions of the random vector $\mathbf{H}$ is still in high stochastic dimension. For instance, the components of $\boldsymbol{\alpha}_{\text {par }}$ are the correlation lengths, the dispersion parameters controlling the statistical fluctuations levels, etc.

\subsection{Prior probabilistic model of model uncertainties}

Let $\left(\Theta^{\prime}, \mathcal{T}^{\prime}, \mathcal{P}^{\prime}\right)$ be another probability space. As explained in [32], the dependent random matrices $\left[\mathbf{M}_{\text {par }}\right],\left[\mathbf{D}_{\text {par }}\right]$ and $\left[\mathbf{K}_{\text {par }}\right]$ are replaced by the dependent random matrices $[\mathbf{M}],[\mathbf{D}]$ and $[\mathbf{K}]$, defined on a probability space $\left(\Theta \times \Theta^{\prime}, \mathcal{T}^{\prime} \otimes \mathcal{T}^{\prime}, \mathcal{P} \otimes \mathcal{P}^{\prime}\right)$, such that, for $A$ denoting $M, D$ or $K$, one has

$$
[\mathbf{A}]=\left[\mathbf{L}_{A}\right]^{T}\left[\mathbf{G}_{A}\right]\left[\mathbf{L}_{A}\right]
$$

in which the probability distributions of the random matrices $\left[\mathbf{G}_{M}\right],\left[\mathbf{G}_{D}\right]$ and $\left[\mathbf{G}_{K}\right]$ defined on $\left(\Theta^{\prime}, \mathcal{T}^{\prime}, \mathcal{P}^{\prime}\right)$, are explicitly given in $[29]$ in the context of the nonparametric probabilistic approach of uncertainties. The nonparametric probabilistic approach of uncertainties is summarized in Appendix A. The probability distributions of the random matrices $\left[\mathbf{G}_{M}\right],\left[\mathbf{G}_{D}\right]$ and $\left[\mathbf{G}_{K}\right]$ depends on the dispersion parameters $\delta_{M}, \delta_{D}, \delta_{K}$ respectively. We introduce the vector $\boldsymbol{\alpha}_{\bmod }$ of the dispersion parameters such that $\boldsymbol{\alpha}_{\bmod }=\left(\delta_{M}, \delta_{D}, \delta_{K}\right)$. The prior probabilistic model of model uncertainties is then completely defined by vector $\boldsymbol{\alpha}_{\text {mod }}$ which has to be identified using experimental responses. For $\theta$ in $\Theta$ and $\theta^{\prime}$ in $\Theta^{\prime}$, the realizations $\left[\mathbf{M}\left(\theta, \theta^{\prime}\right)\right],\left[\mathbf{D}\left(\theta, \theta^{\prime}\right)\right]$ and $\left[\mathbf{K}\left(\theta, \theta^{\prime}\right)\right]$ of the random generalized mass, damping and stiffness matrices $[\mathbf{M}],[\mathbf{D}]$ and $[\mathbf{K}]$ are, if $A$ denotes $M, D$ or $K$, such that

$$
\left[\mathbf{A}\left(\theta, \theta^{\prime}\right)\right]=\left[\mathbf{L}_{A}(\theta)\right]^{T}\left[\mathbf{G}_{A}\left(\theta^{\prime}\right)\right]\left[\mathbf{L}_{A}(\theta)\right] .
$$

The prior probabilistic model of model uncertainties is represented by $3 n(n+1) / 2$ independent random variables (see Appendix A), therefore, its stochastic dimension is high. Nevertheless, the identification of the prior probabilistic model of model uncertainties will be facilitated by the fact that it is completely defined by vector $\boldsymbol{\alpha}_{\bmod }$ which is of small dimension.

4.3 Random responses of the stochastic reduced-order computational model

For all $\omega$ in $B$, for all $\theta$ in $\Theta$ and for all $\theta^{\prime}$ in $\Theta^{\prime}$, the realization $\mathbf{Y}\left(\omega ; \theta, \theta^{\prime}\right)$ of the random response $\mathbf{Y}(\omega)$ of the stochastic reduced-order computational model, is written as

$$
\mathbf{Y}\left(\omega ; \theta, \theta^{\prime}\right)=[\Phi(\mathbf{H}(\theta))] \mathbf{Q}\left(\omega ; \theta, \theta^{\prime}\right),
$$


in which the realization $\mathbf{Q}\left(\omega ; \theta, \theta^{\prime}\right)$ of the random vector $\mathbf{Q}(\omega)$ of the random generalized coordinates, is the solution of the following deterministic reduced-order matrix equation,

$$
\left(-\omega^{2}\left[\mathbf{M}\left(\theta, \theta^{\prime}\right)\right]+i \omega\left[\mathbf{D}\left(\theta, \theta^{\prime}\right)\right]+\left[\mathbf{K}\left(\theta, \theta^{\prime}\right)\right]\right) \mathbf{Q}\left(\omega, \theta, \theta^{\prime}\right)=\mathbf{f}(\omega ; \mathbf{H}(\theta))
$$

With the above construction, it can be proven that $\{\mathbf{Y}(\omega), \omega \in B\}$ is a second-order stochastic process.

\section{Global Identification of the stochastic reduced-order computational model parameters}

The prior probabilistic model of system-parameters uncertainties depends on parameter $\boldsymbol{\alpha}_{\text {par }}$ and the prior probabilistic model of model uncertainties depends on parameter $\boldsymbol{\alpha}_{\text {mod }}$. We propose to simultaneously identify the parameters $\boldsymbol{\alpha}_{\text {par }}$ and $\boldsymbol{\alpha}_{\text {mod }}$ using $\nu_{\exp }$ experimental observations related to the frequency responses corresponding to $\nu_{\text {exp }}$ experimental configurations of the real dynamical system.

For each experimental configuration, the response is measured for $n_{\text {obs }}$ degrees-of-freedom at $n_{\text {freq }}$ frequencies belonging to frequency band $B$. For each frequency $\omega_{i}$ with $i=1, \ldots, n_{\text {freq }}$, we introduce

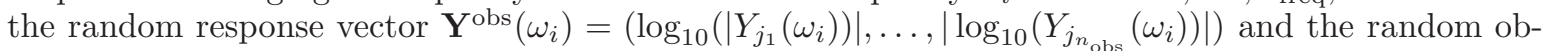
servation vector $\mathbf{Z}^{\mathrm{obs}}=\left(\mathbf{Y}^{\mathrm{obs}}\left(\omega_{1}\right), \ldots, \mathbf{Y}^{\mathrm{obs}}\left(\omega_{n_{\text {freq }}}\right)\right)$ with values in $\mathbb{R}^{n_{\mathrm{obs}} \times n_{\text {freq }}}$. The corresponding $\nu_{\exp }$ experimental observation vectors are denoted by $\mathbf{z}_{1}^{\exp }, \ldots, \mathbf{z}_{\nu_{\exp }}^{\exp }$.

The parameters $\boldsymbol{\alpha}_{\text {par }}$ and $\boldsymbol{\alpha}_{\bmod }$ are identified using the maximum likelihood method with the experimental responses $[27 ; 33 ; 37]$. Let $\mathcal{C}_{\text {par }}$ and $\mathcal{C}_{\text {mod }}$ be the admissible spaces for parameters $\boldsymbol{\alpha}_{\text {par }}$ and $\boldsymbol{\alpha}_{\text {mod. }}$. The optimal values $\boldsymbol{\alpha}_{\text {par }}^{\text {opt }}$ and $\boldsymbol{\alpha}_{\text {mod }}^{\text {opt }}$ are solution of the following optimization problem

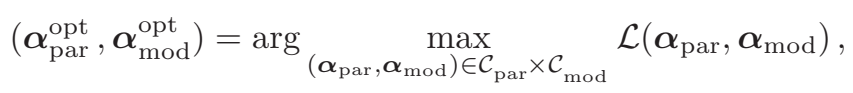

with

$$
\mathcal{L}\left(\boldsymbol{\alpha}_{\text {par }}, \boldsymbol{\alpha}_{\text {mod }}\right)=\sum_{\ell=1}^{\nu_{\text {exp }}} \log \left(p_{\text {zobs }_{\text {obs }}}\left(\mathbf{z}_{\ell}^{\text {exp }} ; \boldsymbol{\alpha}_{\text {par }}, \boldsymbol{\alpha}_{\text {mod }}\right)\right.
$$

where $p_{\mathbf{z}^{\text {obs }}}\left(\mathbf{z} ; \boldsymbol{\alpha}_{\text {par }}, \boldsymbol{\alpha}_{\text {mod }}\right)$ is the probability density function of the random vector $\mathbf{Z}^{\text {obs }}$. As proposed in [32], this probability density function is estimated using a statistical reduction of the random vector $\mathbf{Z}^{\text {obs }}$.

\section{Application}

As explained in Section 1, the paper presents a methodology for identifying random parameters of a linear dynamical system in presence of modeling errors. The random parameters are not a few real random variables, but are two random fields. The main objective of the application is to demonstrate the capability of the proposed methodology using a case for which there are modeling errors and for which the statistical fluctuations of the random fields have significant influences on the stochastic dynamical responses. Typically, this kind of situation can be found in micromechanics of heterogeneous composites for which a mesoscale modeling is performed. This is the reason why a three-dimensional slender elastic body has been chosen for generating the simulated experiments because, it is known that the use of the beam theory for modeling such a system collapses when frequency increases. With such an application, modeling errors exist and then, the validation of the identification procedure of random fields is carried out in a correct framework. Since the response is analyzed on a large frequency band and not only for the first two or three elastic modes, the application proposed is representative of more complex mechanical systems with respect to the identification procedure in the area of linear structural dynamics with many elastic modes. Consequently, the simulated experiments of the mechanical system considered allow us to carry out a clear validation of the identification procedure for linear dynamical systems for which the random fields properties have a significant impact on the random responses and for which the computational model of the dynamical system exhibits modeling errors. 


\subsection{Real system and generation of random experimental responses}

The real system is a slender cylindrical bounded domain $\Omega^{\mathrm{exp}}$, with a rectangular section, given in a cartesian coordinate system $\left(O x_{1} x_{2} x_{3}\right)$. This real system has length $10 \mathrm{~m}$, width $1.1 \mathrm{~m}$ and height1.6 m. The two end sections are located at $x_{1}=0$ and $x_{1}=10$. The origin $O$ is in the corner of the end section and $O x_{1}$ is parallel to the cylinder axis. Axis $O x_{2}$ is the transversal axis along the width and $O x_{3}$ is the lateral axis along the height (see Fig.1). The neutral line has for equation $\left\{0 \leq x_{1} \leq 10 ; x_{2}=0.550 ; x_{3}=0.800\right\}$. Concerning the boundary condition, the displacement fields is zero on the two end sections. The frequency band of analysis is $B=[0,1200] \mathrm{Hz}$. The domain $\Omega^{\exp }$ of the real system is made up of two subdomains $\Omega_{1}^{\exp }$ and $\Omega_{2}^{\exp }$. Subdomain $\Omega_{1}^{\exp }$ is defined by $\left\{0 \leq x_{1} \leq 3.75\right\}$ and subdomain $\Omega_{2}^{\exp }$ is defined by $\left\{3.75 \leq x_{1} \leq 10\right\}$. In this model, the material is isotropic with homogeneous mass density $1600 \mathrm{Kg} / \mathrm{m}^{3}$. The damping rate is 0.01 . The bulk modulus and the shear modulus are variable following $x_{1}$ direction and are modeled by random fields. In $\Omega_{1}^{\exp }$, the bulk modulus $\left\{x_{1} \mapsto K_{1}^{\exp }\left(x_{1}\right)\right\}$ is a Gamma random field with mean value $5.56 \times 10^{9} \mathrm{~N} / \mathrm{m}^{2}$, coefficient of variation 0.2 and correlation length $0.375 \mathrm{~m}$ and the shear modulus $\left\{x_{1} \mapsto G_{1}^{\exp }\left(x_{1}\right)\right\}$ is a Gamma random field with mean value $4.17 \times 10^{9} \mathrm{~N} / \mathrm{m}^{2}$, coefficient of variation 0.09 and correlation length $0.375 \mathrm{~m}$. In $\Omega_{2}^{\exp }$, the bulk modulus $\left\{x_{1} \mapsto K_{2}^{\exp }\left(x_{1}\right)\right\}$ is a Gamma random field with mean value $6.67 \times 10^{9} \mathrm{~N} / \mathrm{m}^{2}$, coefficient of variation 0.2 and correlation length $0.625 \mathrm{~m}$ and the shear modulus $\left\{x_{1} \mapsto G_{2}^{\exp }\left(x_{1}\right)\right\}$ is a Gamma random field with mean value $5.0 \times 10^{9} \mathrm{~N} / \mathrm{m}^{2}$, coefficient of variation 0.09 and correlation length $0.625 \mathrm{~m}$. Random fields $K_{1}^{\exp }, G_{1}^{\exp }, K_{2}^{\exp }$ and $G_{2}^{\text {exp }}$ are statistically independent. The reasons for such a construction of random fields $K_{1}^{\exp }, G_{1}^{\exp }, K_{2}^{\exp }$ and $G_{2}^{\text {exp }}$ can be found in [14] in which the statistically dependence between the components of the random elasticity tensors that exhibit some given material symmetries is characterized.

With such a construction, the random tensor related to the elastic part of the elasticity tensor is written as $a_{h \ell j k}\left(x_{1}\right)=K_{1}^{\exp }\left(x_{1}\right) \delta_{h \ell} \delta_{j k}+G_{1}^{\exp }\left(x_{1}\right)\left(\left(\delta_{h j} \delta_{\ell k}+\delta_{h k} \delta_{\ell j}\right)-2 \delta_{h \ell} \delta_{j k} / 3\right)$ in $\Omega_{1}^{\exp }$ and $a_{h \ell j k}\left(x_{1}\right)=$ $K_{2}^{\exp }\left(x_{1}\right) \delta_{h \ell} \delta_{j k}+G_{2}^{\exp }\left(x_{1}\right)\left(\left(\delta_{h j} \delta_{\ell k}+\delta_{h k} \delta_{\ell j}\right)-2 \delta_{h \ell} \delta_{j k} / 3\right)$ in $\Omega_{2}^{\exp }$, in which $\delta_{i j}$ is the Kronecker symbol. The tensor related to the dissipative part of the elasticity tensor $b_{h \ell j k}(\mathbf{x})$ is deterministic.

The random Young modulus fields in $\Omega_{1}^{\exp }$ and in $\Omega_{2}^{\exp }$ are written as $E_{1}^{\exp }=9 K_{1}^{\exp } G_{1}^{\exp } /\left(3 K_{1}^{\exp }+\right.$ $\left.G_{1}^{\exp }\right)$ and $E_{2}^{\exp }=9 K_{2}^{\exp } G_{2}^{\exp } /\left(3 K_{2}^{\exp }+G_{2}^{\exp }\right)$, and the Poisson ratio fields are written as $N_{1}^{\exp }=$ $0.5 \times\left(3 K_{1}^{\text {exp }}-2 G_{1}^{\text {exp }}\right) /\left(3 K_{1}^{\text {exp }}+G_{1}^{\text {exp }}\right)$ and $N_{2}^{\text {exp }}=0.5 \times\left(3 K_{2}^{\text {exp }}-2 G_{2}^{\text {exp }}\right) /\left(3 K_{2}^{\text {exp }}+G_{2}^{\text {exp }}\right)$. Random fields $E_{1}^{\exp }, E_{2}^{\text {exp }}, N_{1}^{\text {exp }}$ and $N_{2}^{\text {exp }}$ are statistically dependent. Using a Karhunen-Loève decomposition, each random field $K_{1}^{\exp }, G_{1}^{\exp }, K_{2}^{\exp }$ and $G_{2}^{\exp }$ can be expressed by 30 (for an accuracy $10^{-2}$ ) uncorrelated random variables. Therefore, the stochastic dimension concerning the system-parameter uncertainties is 120 which is a very high stochastic dimension with respect to the state-of-the art for the stochastic solvers based on the stochastic spectral methods.

The external load is a point load applied at the point $\left(x_{1}=4.250, x_{2}=1.100, x_{3}=0.800\right)$ and its Fourier transform is a vector valued function $\nu \mapsto\left(0,-\mathbb{1}_{B}(\nu), 0\right)$ in which $\mathbb{1}_{B}(\nu)=1$ if $\nu$ belongs to $B$ and $\mathbb{1}_{B}(\nu)=0$ otherwise. The finite element mesh of the real system is made up of 5,760 three-dimensional 8-nodes solid elements (see Fig.1) and the finite element model has 25, 095 degreesof-freedom. We are interested in the random transversal displacement along $O x_{2}$ in the plane $O x_{1} x_{2}$ of the neutral line at four observation points $P_{1}, P_{2}, P_{3}$ and $P_{4}$ belonging to the neutral line for which $x_{1}$ are $1.875,3.125,4.250$ and $6.375 \mathrm{~m}$. We then have $n_{\mathrm{obs}}=4$.

A total of $\nu_{\exp }=200$ experimental configurations have been simulated. For each experimental configuration $\ell$, the observation vector $\mathbf{z}_{\ell}^{\text {exp }}$ is calculated using the modal analysis for which the number of elastic modes is 180 that is sufficient to ensure a good mean-square convergence in the frequency band of analysis $B=[0,1200] \mathrm{Hz}$.

\subsection{Nominal computational model and reduced-order nominal computational model}

The nominal computational model is made up of a damped Timoshenko elastic beam with length $L_{1}=10 \mathrm{~m}$, clamped at $x_{1}=0$ and $x_{1}=10$. The section of the beam is the same as the section of the real system presented in Section 6.1. The domain $\Omega$ of the beam is still made up of two subdomains $\Omega_{1}$ and $\Omega_{2}$. Subdomain $\Omega_{1}$ is defined by $\left\{0 \leq x_{1} \leq 3.75\right\}$ and subdomain $\Omega_{2}$ is defined by $\left\{3.75 \leq x_{1} \leq 10\right\}$. In this nominal computational model, the material is isotropic with homogeneous mass density $1600 \mathrm{Kg} / \mathrm{m}^{3}$. The damping rate is 0.01 . The bulk modulus and the shear modulus are 


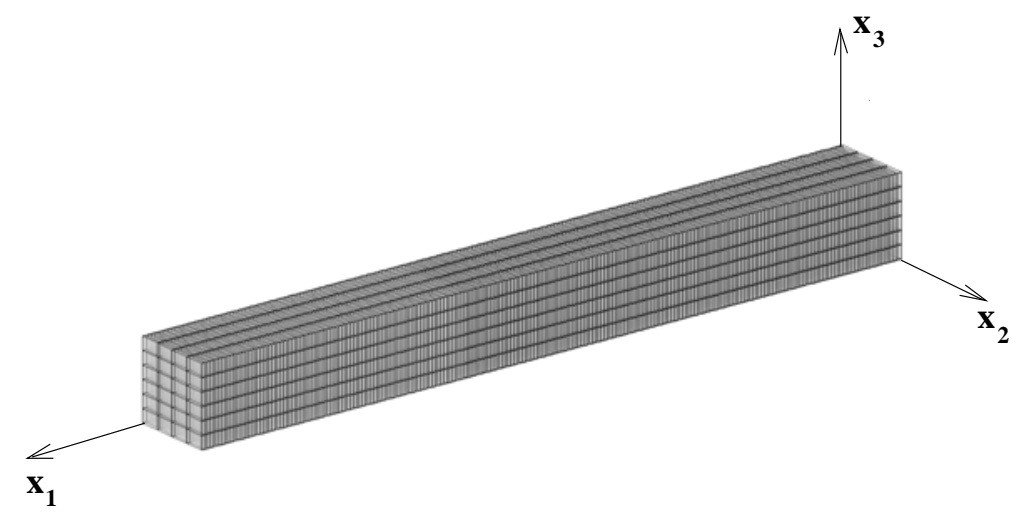

Fig. 1 Domain $\Omega^{\exp }$ of the real system, coordinate system and finite element mesh of the real system.

variable following $x_{1}$ direction. In $\Omega_{1}$, the bulk modulus $\left\{x_{1} \mapsto k_{1}\left(x_{1}\right)\right\}$ is $5.56 \times 10^{9} \mathrm{~N} / \mathrm{m}^{2}$ and the shear modulus $\left\{x_{1} \mapsto g_{1}\left(x_{1}\right)\right\}$ is $4.17 \times 10^{9} \mathrm{~N} / \mathrm{m}^{2}$. In $\Omega_{2}$, the bulk modulus $\left\{x_{1} \mapsto k_{2}\left(x_{1}\right)\right\}$ is $6.67 \times 10^{9} \mathrm{~N} / \mathrm{m}^{2}$ and the shear modulus $\left\{x_{1} \mapsto g_{2}\left(x_{1}\right)\right\}$ is $5.0 \times 10^{9} \mathrm{~N} / \mathrm{m}^{2}$. The external load is a point load applied at the point $x_{1}=4.250$ and its Fourier transform is a vector valued function $\nu \mapsto\left(0,-\mathbb{1}_{B}(\nu), 0\right)$. The finite element mesh is made up of 240 Timoshenko beam elements and the finite element model has 1,434 degrees-of-freedom. It should be noted that the finite elements used do not take into account the rotational inertia. Consequently, the error on the kinetic energy is larger than if it had been taken into account. Therefore, the dispersion parameter, $\delta_{M}$, which controls the level of modeling errors will be larger. This choice is intentional in order to generate modeling errors which allow us to validate the identification procedure of the random fields in presence of significant modeling errors.

The frequency response functions (FRF) are calculated using the reduced-order nominal computational model presented in Section 3.2 with $n=61$. In the frequency band $B=[0,1200] \mathrm{Hz}$, there are 51 elastic modes and in the frequency band $[1200,1400] \mathrm{Hz}$, there are 9 elastic modes. Figures 2 to 5 display the comparisons between the experimental FRF and the FRF computed with the reducedorder nominal computational model for the transversal displacements at observation points $P_{1}, P_{2}, P_{3}$ and $P_{4}$. The objective of the comparison is to analyze the robustness of the reduced-order nominal computational model as a function of the frequency and to highlight the need to construct a stochastic reduced-order computational model to improve the robustness Concerning the experimental FRF, the figures display the confidence regions which have been estimated with a probability level $P_{c}=0.95$. Figures 2 to 5 clearly show that the low-frequency band is $[0,550] \mathrm{Hz}$ while the medium-frequency band is $[550,1200] \mathrm{Hz}$. The very low-frequency band can be identified as $[0,180] \mathrm{Hz}$ (the first three elastic modes) for which the frequency responses are very robust with respect to uncertainties while the frequency responses in the low-frequency-band $[180,550] \mathrm{Hz}$ are sensitive to uncertainties. In the medium-frequency band, the responses strongly depends on uncertainties.

\subsection{Stochastic reduced-order computational model}

The stochastic reduced-order computational model is constructed as explained in Section 4.

\subsubsection{System-parameters uncertainties and random responses}

Concerning system-parameters uncertainties, the deterministic bulk moduli $k_{1}, k_{2}$ and shear moduli $g_{1}$, $g_{2}$ are modeled by the independent random fields $K_{1}, K_{2}, G_{1}$ and $G_{2}$. The prior probabilistic models are defined as follows. Let $\beta>0$ be an updating parameter. The bulk modulus $\left\{x_{1} \mapsto K_{1}\left(x_{1}\right)\right\}$ is a Gamma random field with mean value $\beta \times 5.56 \times 10^{9} \mathrm{~N} / \mathrm{m}^{2}$, coefficient of variation 0.2 and correlation length $0.375 \mathrm{~m}$. The shear modulus $\left\{x_{1} \mapsto G_{1}\left(x_{1}\right)\right\}$ is a Gamma random field with mean value $4.17 \times 10^{9} \mathrm{~N} / \mathrm{m}^{2}$, coefficient of variation 0.09 and correlation length $0.375 \mathrm{~m}$. The bulk modulus $\left\{x_{1} \mapsto K_{2}\left(x_{1}\right)\right\}$ is a Gamma random field with mean value $\beta \times 6.67 \times 10^{9} \mathrm{~N} / \mathrm{m}^{2}$, coefficient of variation 0.2 and correlation length $0.625 \mathrm{~m}$ and the shear modulus $\left\{x_{1} \mapsto G_{2}\left(x_{1}\right)\right\}$ is a Gamma random field with mean value 


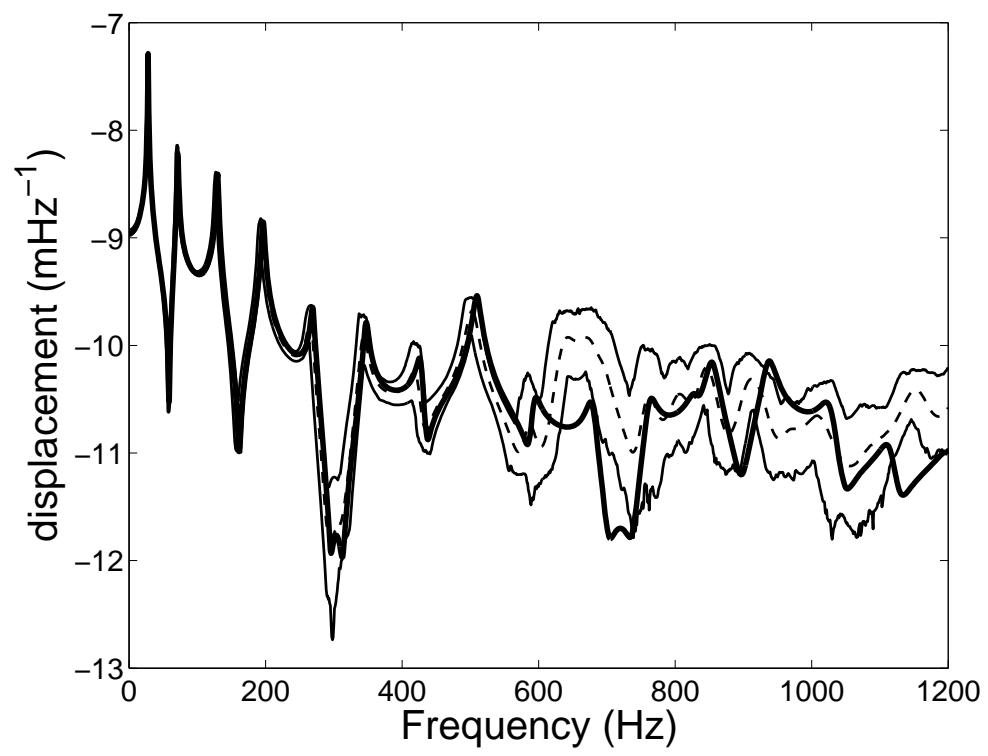

Fig. 2 FRF computed with the reduced-order nominal computational model (thick solid line). Experimental FRF: Confidence region (upper and lower thin solid lines) and mean response (thin dashed line). Observation point $P_{1}$.

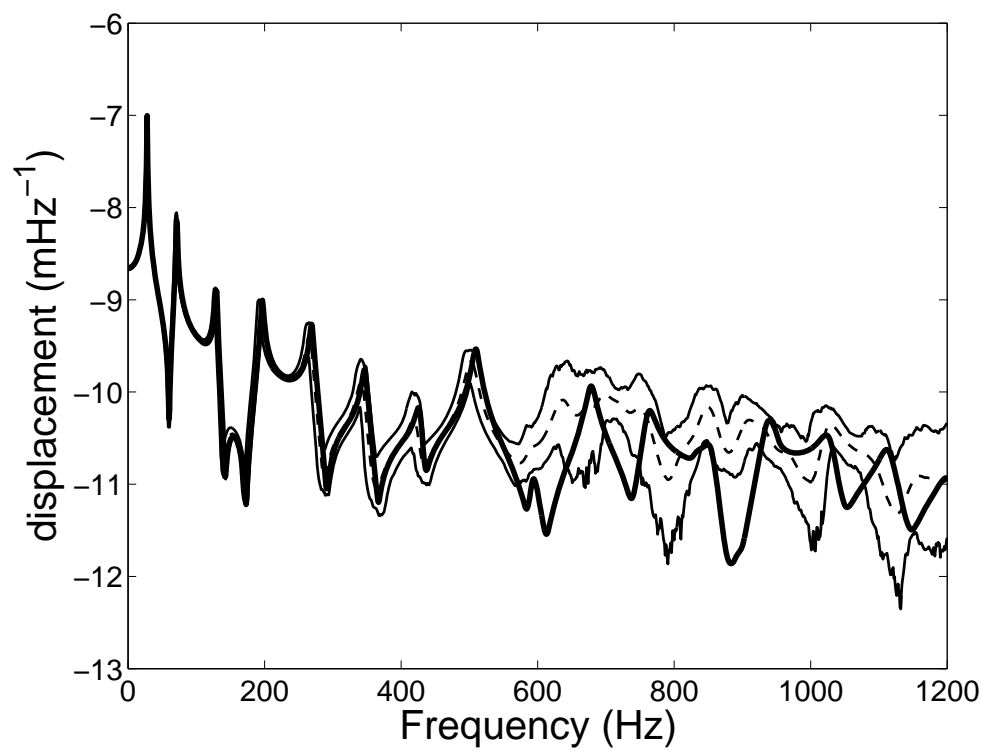

Fig. 3 FRF computed with the reduced-order nominal computational model (thick solid line). Experimental FRF: Confidence region (upper and lower thin solid lines) and mean response (thin dashed line). Observation point $P_{2}$.

$5.0 \times 10^{9} \mathrm{~N} / \mathrm{m}^{2}$, coefficient of variation 0.09 and correlation length $0.625 \mathrm{~m}$. Random fields $K_{1}, G_{1}$, $K_{2}$ and $G_{2}$ are independent. The prior probabilistic models of system-parameters uncertainties are completely defined and depend on the parameter $\beta$. Consequently, the parameter $\boldsymbol{\alpha}_{\text {par }}$ introduced in Section 4.1 is such that $\boldsymbol{\alpha}_{\mathrm{par}}=(\beta)$. It should be noted that for $\beta=1$, the random fields of the stochastic reduced-order computational model are identical to the random fields we have used to generate the experimental configurations. These random fields are discretized in the 240 integration points (reduced integration, see [15]).

For observation points $P_{1}, P_{2}, P_{3}$ and $P_{4}$ and for $\beta=1$, the random frequency response functions are displayed in Figs. 6 to 9. The confidence regions are estimated with a probability level $P_{c}=0.95$ 


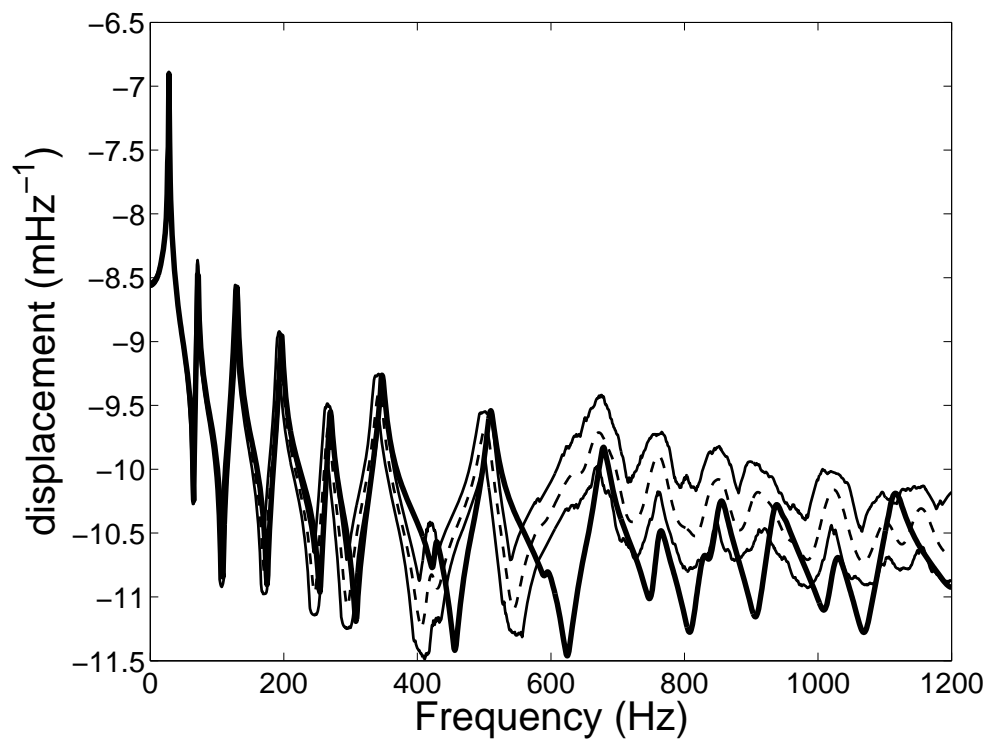

Fig. 4 FRF computed with the reduced-order nominal computational model (thick solid line). Experimental FRF: Confidence region (upper and lower thin solid lines) and mean response (thin dashed line). Observation point $P_{3}$.

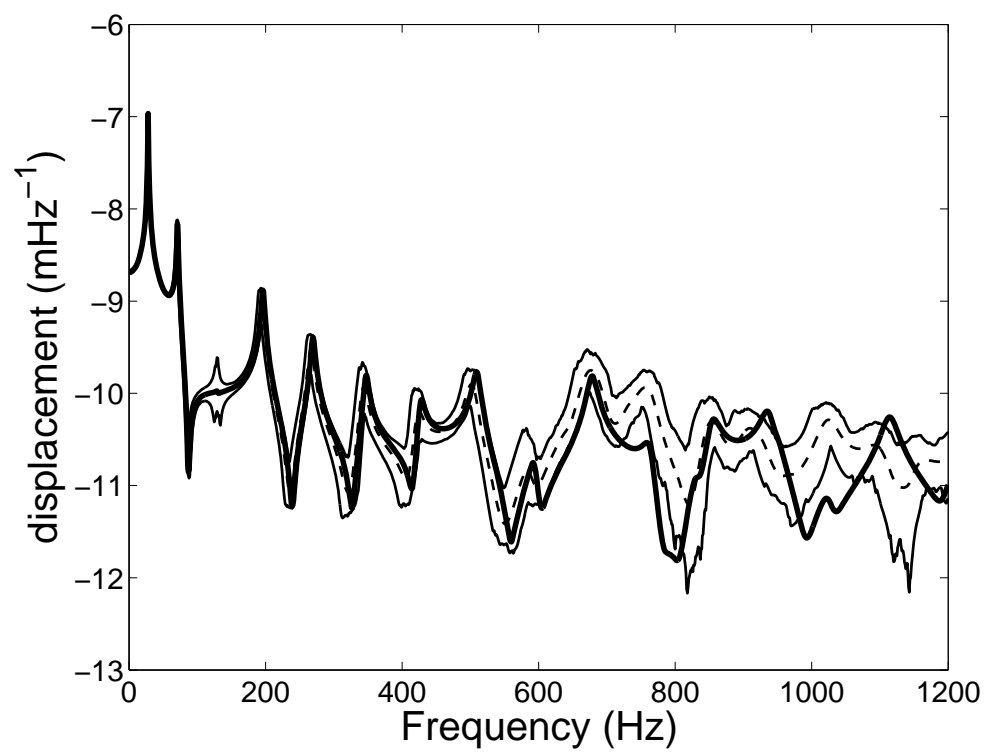

Fig. 5 FRF computed with the reduced-order nominal computational model (thick solid line). Experimental FRF: Confidence region (upper and lower thin solid lines) and mean response (thin dashed line). Observation point $P_{4}$.

with 100, 000 Monte Carlo simulations.

Figures 6 to 9 clearly show that the introduction of the probabilistic models of the system-parameters uncertainties improves the predictions in the low-frequency band $[0,550] \mathrm{Hz}$ (the confidence regions predicted with the stochastic reduced-order computational model are close to the confidence regions of the experiments). However, the predictions are not really improved in the medium-frequency band $[550,1200] \mathrm{Hz}$. The confidence regions of the experiments are not similar or are not included in the confidence regions predicted with the stochastic reduced-order computational model. These results confirm that the probabilistic parametric approach of uncertainties has not the capability to take into account modeling errors and that this type of model uncertainties must be taken into account to 
improve the quality of the predictions in the high part of the low-frequency band and in the mediumfrequency band.

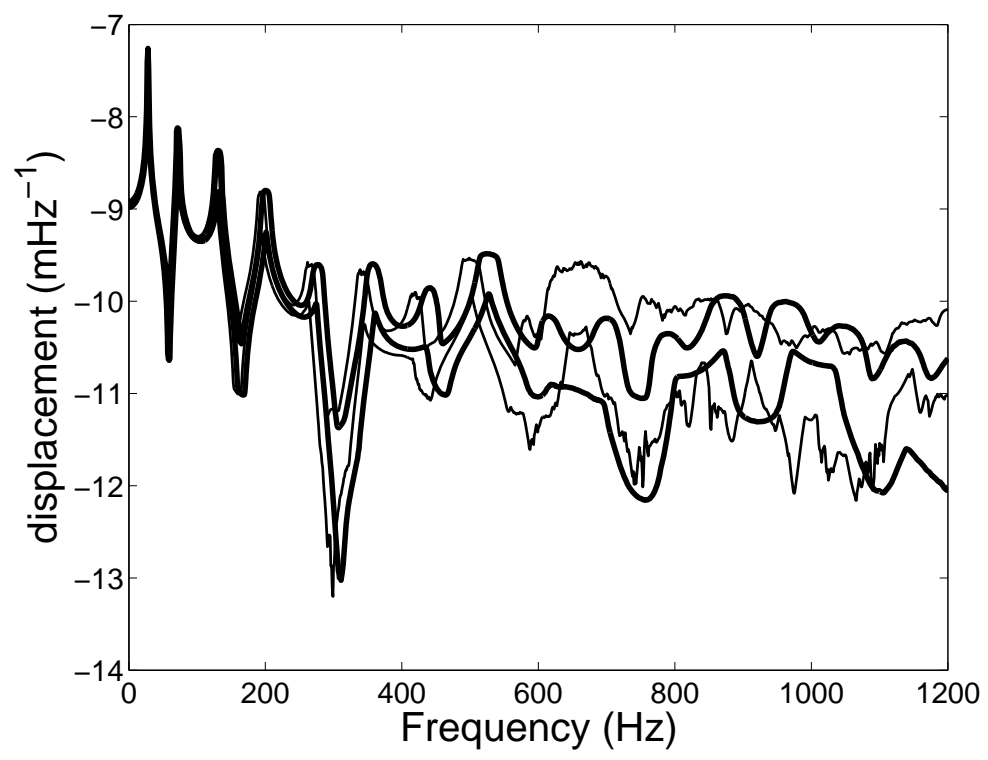

Fig. 6 Random FRF computed with the stochastic reduced-order computational model only with systemparameters uncertainties: Confidence region (upper and lower thick lines). Experimental FRF: Confidence region (upper and lower thin lines). Observation point $P_{1}$.

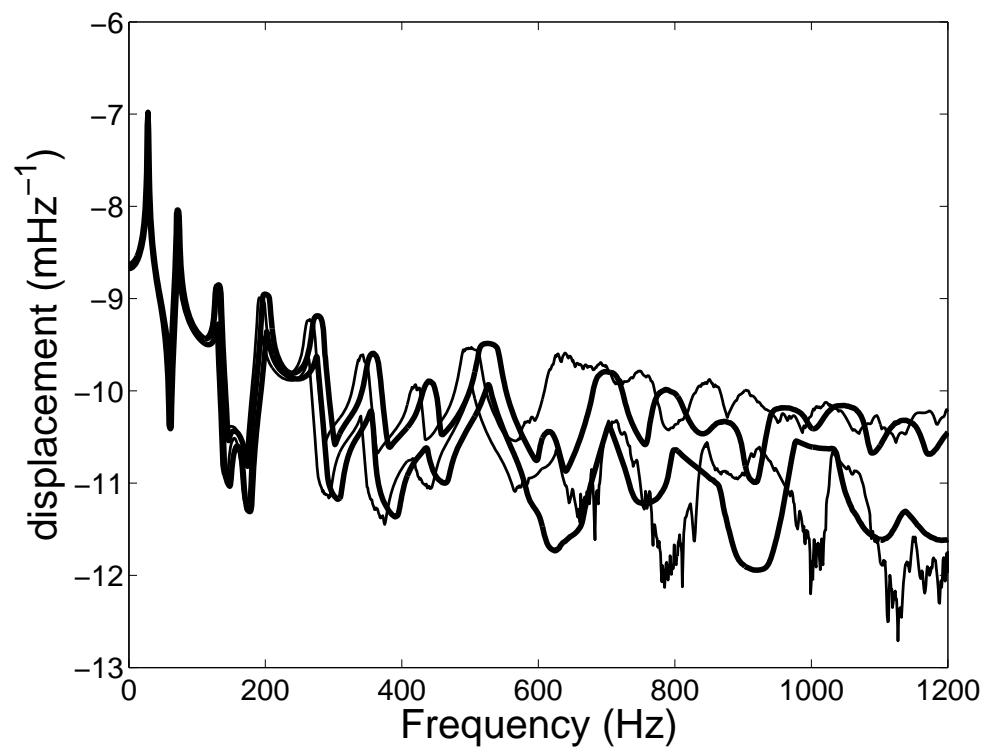

Fig. 7 Random FRF computed with the stochastic reduced-order computational model only with systemparameters uncertainties: Confidence region (upper and lower thick lines). Experimental FRF: Confidence region (upper and lower thin lines). Observation point $P_{2}$. 


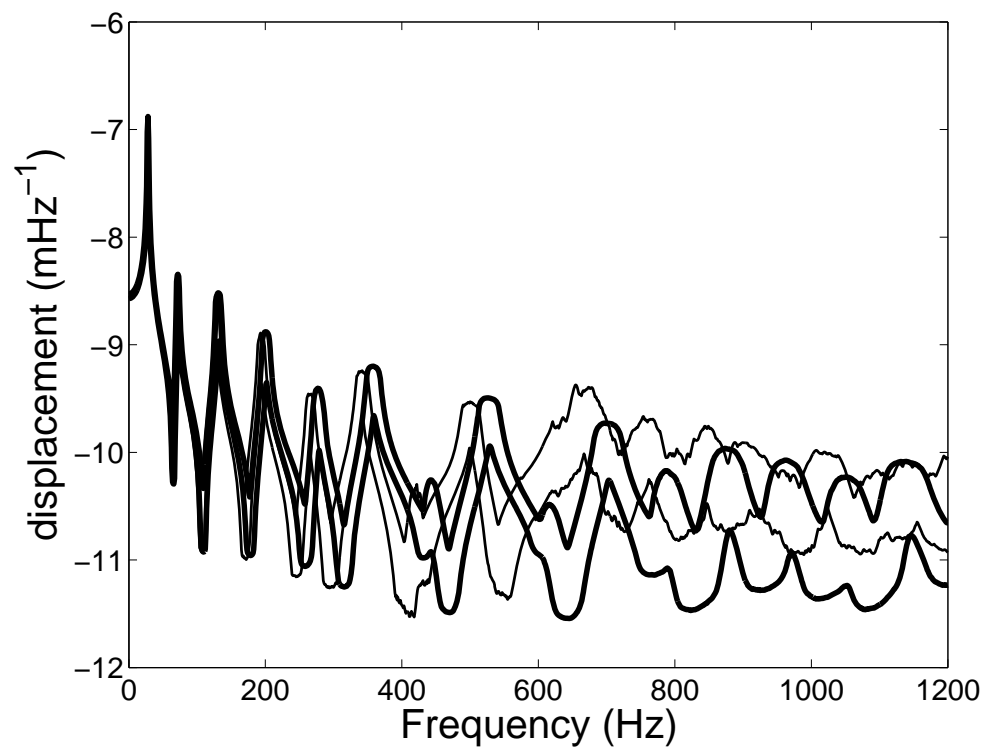

Fig. 8 Random FRF computed with the stochastic reduced-order computational model only with systemparameters uncertainties: Confidence region (upper and lower thick lines). Experimental FRF: Confidence region (upper and lower thin lines). Observation point $P_{3}$.

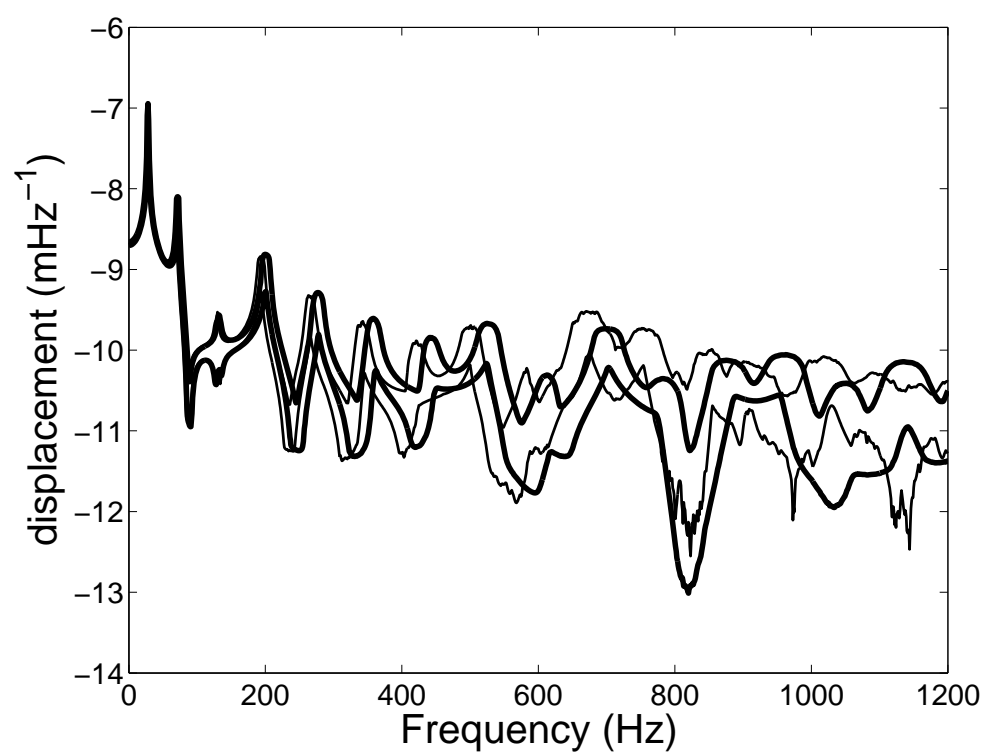

Fig. 9 Random FRF computed with the stochastic reduced-order computational model only with systemparameters uncertainties: Confidence region (upper and lower thick lines). Experimental FRF: Confidence region (upper and lower thin lines). Observation point $P_{4}$.

\subsubsection{Model uncertainties and random responses}

Concerning model uncertainties, it is assumed that the damping is certain (no uncertainties). Therefore, the stochastic modeling of uncertainties due to modeling errors is constructed for the mass and stiffness matrices only. The two parameters which must be identified are $\delta_{M}$ and $\delta_{K}$ and parameter $\boldsymbol{\alpha}_{\bmod }$ introduced in Section 4.2 is such that $\boldsymbol{\alpha}_{\bmod }=\left(\delta_{M}, \delta_{K}\right)$. 
6.3.3 Global identification of the stochastic reduced-order computational model

We use the methodology presented in Section 5 for identifying the parameters $\boldsymbol{\alpha}_{\text {par }}$ and $\boldsymbol{\alpha}_{\text {mod }}$. The optimization problem defined by Eq. (14) is solved using the trial method. The probability density function $p_{\text {zobs }^{\text {obs }}}\left(\mathbf{z}_{\ell}^{\exp } ; \boldsymbol{\alpha}_{\mathrm{par}}, \boldsymbol{\alpha}_{\mathrm{mod}}\right)$ is estimated with 10,000 realizations of the random responses. The optimal values are $\boldsymbol{\alpha}_{\mathrm{par}}^{\mathrm{opt}}=(0.7)$ and $\boldsymbol{\alpha}_{\text {mod }}^{\mathrm{opt}}=(0.5,0.2)$. The likelihood functions $\beta \mapsto \mathcal{L}\left(\beta,\left(\delta_{M}^{\mathrm{opt}}, \delta_{K}^{\mathrm{opt}}\right)\right)$ and $\left(\delta_{M}, \delta_{K}\right) \mapsto \mathcal{L}\left(\beta^{\text {opt }},\left(\delta_{M}, \delta_{K}\right)\right)$ are plotted in Figs. 10 and 11 . It can be viewed that $\delta_{M}^{\text {opt }}$ is larger than $\delta_{K}^{\text {opt }}$. For a Timoshenko beam model, the errors on the elastic energy grow more slowly than the errors on the kinetic energy when the frequency increases, especially for the medium-frequency band. It should be noted that the Timoshenko beam model we have used does not take into account the rotation inertia of the beam section. This induces additional kinetic energy errors (then mass matrix errors). It should be noted that for each value of $\left(\boldsymbol{\alpha}_{\text {par }}, \boldsymbol{\alpha}_{\text {mod }}\right)$, we have simulated 10,000 realizations of the random responses, that could seem as prohibitive if a very large computational model was considered. Using less simulations (1,000 realizations) provides the same results for $\boldsymbol{\alpha}_{\text {par }}^{\mathrm{opt}}$ and $\boldsymbol{\alpha}_{\mathrm{mod}}^{\mathrm{opt}}$, but the estimated likelihood function is a little less smooth. Therefore, the identification method can be applied using less than 10,000 realizations for each value of ( $\left.\boldsymbol{\alpha}_{\text {par }}, \boldsymbol{\alpha}_{\bmod }\right)$.

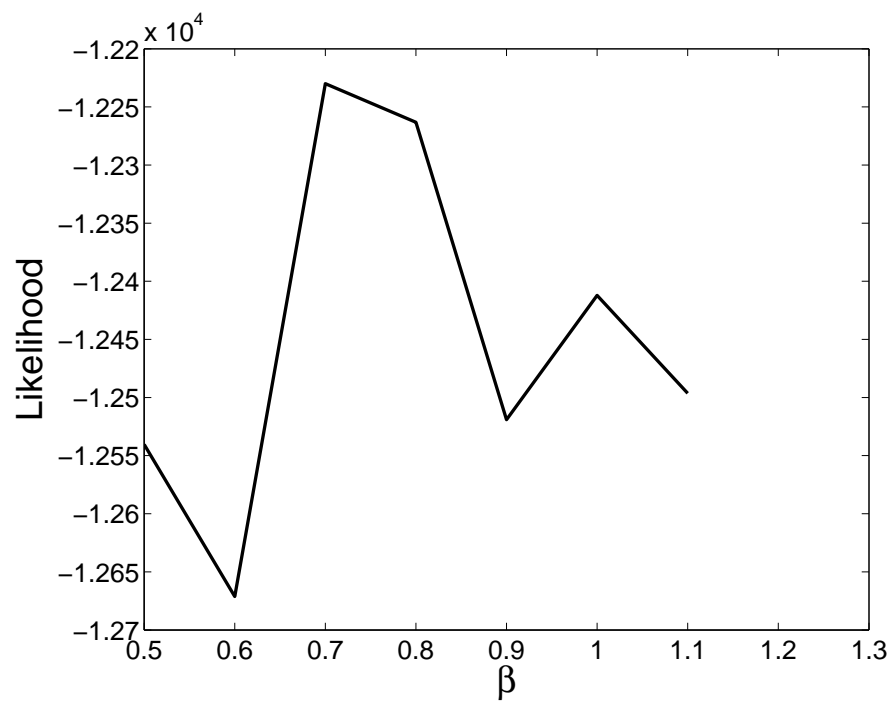

Fig. 10 Graph of function $\beta \mapsto \mathcal{L}\left(\beta,\left(\delta_{M}^{\mathrm{opt}}, \delta_{K}^{\mathrm{opt}}\right)\right)$.

6.3.4 Random frequency response functions predicted with the optimal stochastic reduced-order computational model

For $\boldsymbol{\alpha}_{\text {par }}=\boldsymbol{\alpha}_{\text {par }}^{\text {opt }}$ and $\boldsymbol{\alpha}_{\text {mod }}=\boldsymbol{\alpha}_{\text {mod }}^{\text {opt }}$, the random frequency response functions for observation points $P_{1}$, $P_{2}, P_{3}$ and $P_{4}$ are displayed in Figs. 12 to 15. The confidence regions are estimated with a probability level $P_{c}=0.95$ with 100, 000 Monte Carlo simulations. In the low-frequency band, it can be seen that the confidence regions of the experimental responses are included in the confidence regions of the random responses calculated with the optimal stochastic reduced-order computational model. In the medium-frequency band, the confidence regions of the experimental responses are, for some frequencies, not included in the confidence regions of the random responses calculated with the optimal stochastic reduced-order computational model. This corresponds to the fact that the confidence regions have been estimated with the probability level $P_{c}=0.95$. 


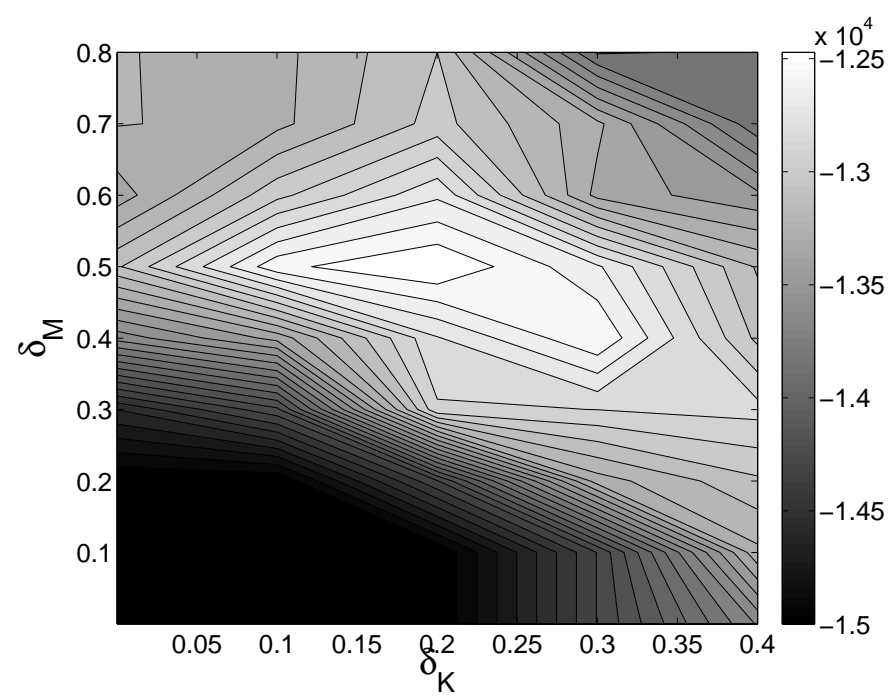

Fig. 11 Graph of function $\left(\delta_{M}, \delta_{K}\right) \mapsto \mathcal{L}\left(\beta^{\mathrm{opt}},\left(\delta_{M}, \delta_{K}\right)\right)$.

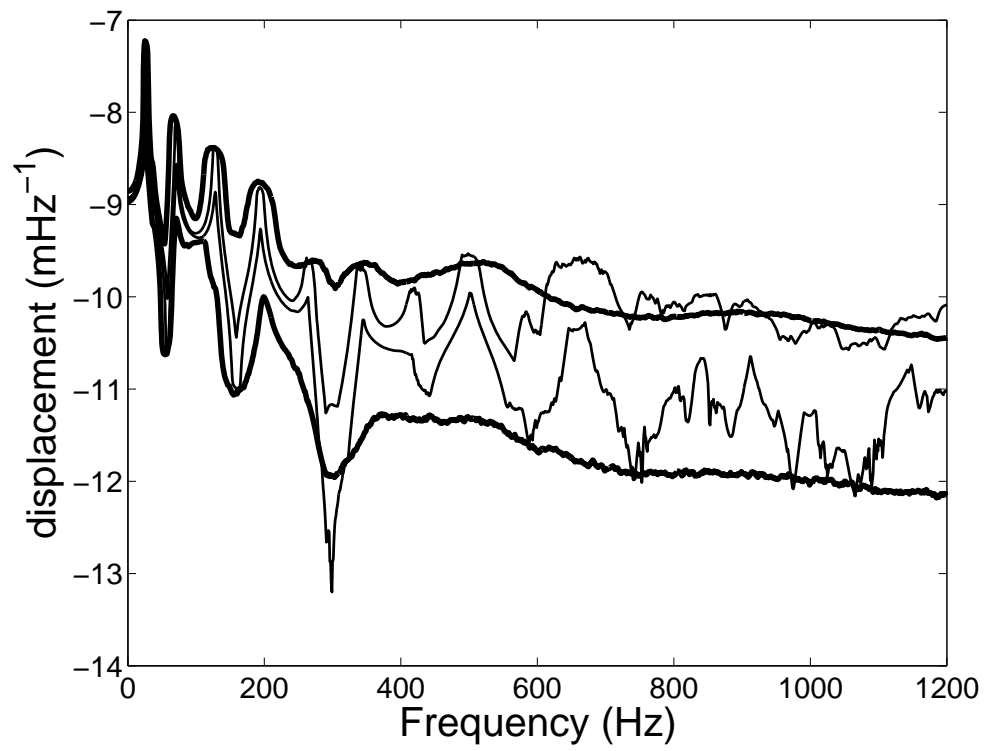

Fig. 12 Random FRF calculated with the optimal stochastic reduced-order computational model: Confidence region (upper and lower thick lines). Experimental FRF: Confidence region (upper and lower thin lines). Observation point $P_{1}$.

\section{Conclusions}

A methodology adapted to the high stochastic dimension has been presented for statistical inverse problems related to uncertain computational models in structural dynamics. This methodology consists (1) in constructing a prior stochastic model of uncertainties described with a small number of parameters, (2) in using a stochastic solver adapted to the high stochastic dimension and (3) in identifying an optimal prior stochastic model using the maximum likelihood method, the stochastic computational model and experimental responses. This methodology has been validated in high stochastic dimension through a numerical application and can be applied to complex real dynamical structures.

For the application, the identified mass dispersion parameter is larger than the identified stiffness dispersion parameter. This result is in agreement with the fact that for a Timoshenko beam modeling, the errors on the elastic energy grow more slowly than the errors on the kinetic energy when the 


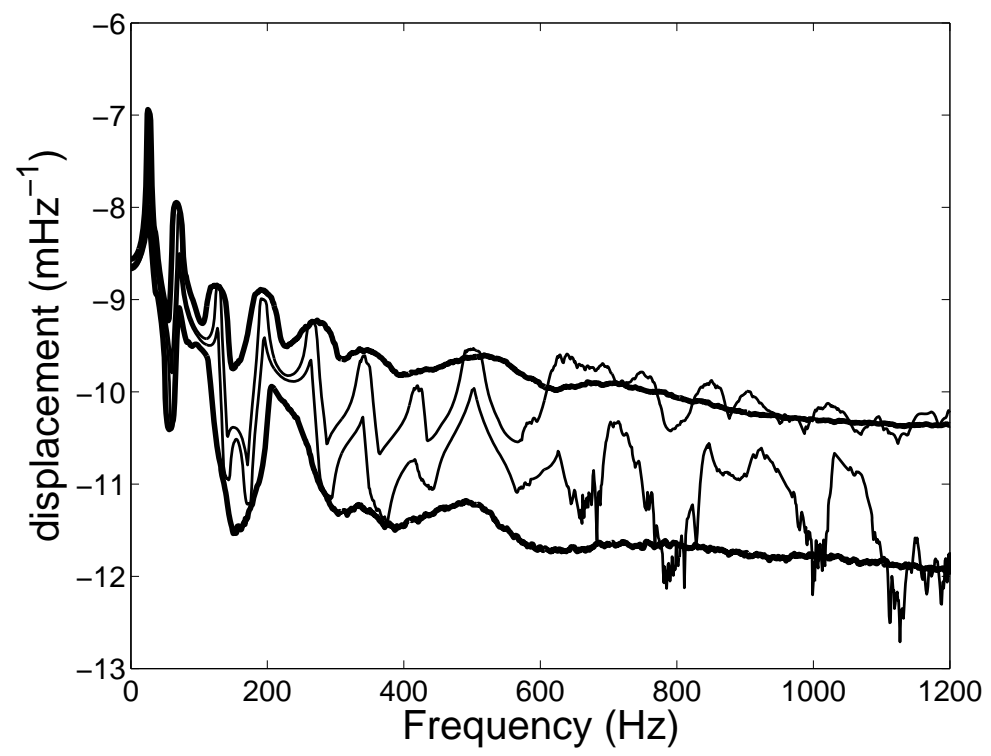

Fig. 13 Random FRF calculated with the optimal stochastic reduced-order computational model: Confidence region (upper and lower thick lines). Experimental FRF: Confidence region (upper and lower thin lines). Observation point $P_{2}$.

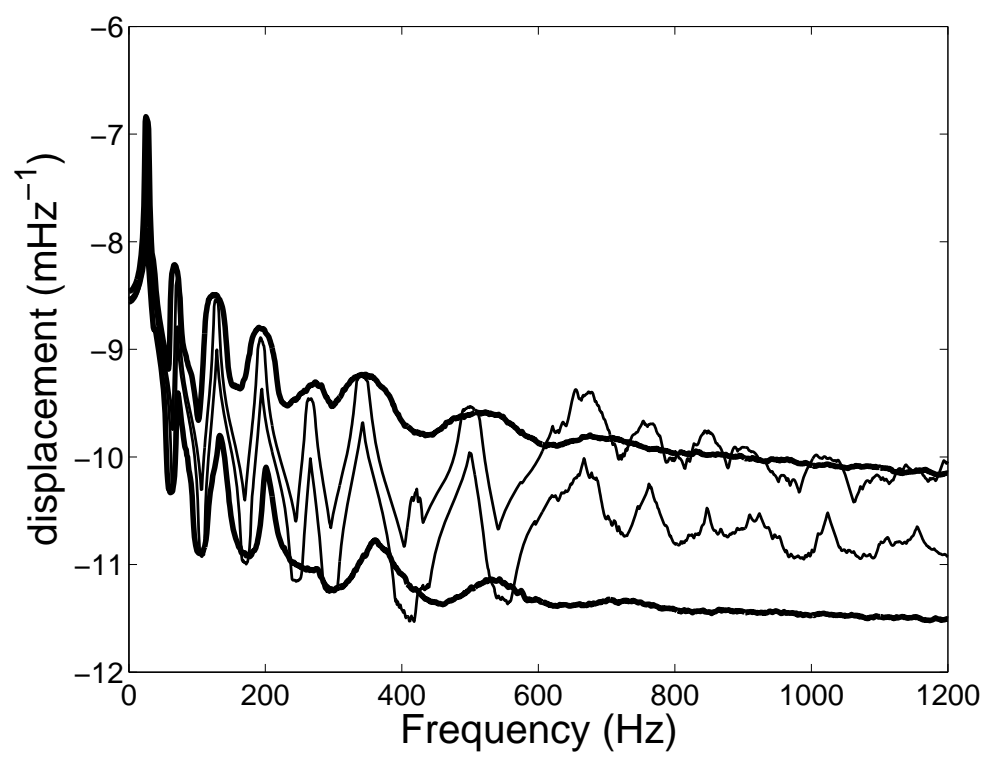

Fig. 14 Random FRF calculated with the optimal stochastic reduced-order computational model: Confidence region (upper and lower thick lines). Experimental FRF: Confidence region (upper and lower thin lines). Observation point $P_{3}$.

frequency increases, especially in the medium-frequency range for which the kinetic energy errors can be important. For the low- and medium-frequency ranges, the random responses are predicted using the stochastic reduced-order computational model with the optimal prior stochastic models of uncertainties. It should be noted that the implementation of a prior probabilistic model of modeling errors allows the validity of the predictions of the computational model with random fields properties to be extended to the medium-frequency range which is very sensitive to modeling errors. A validation of the proposed methodology has been performed using simulated experiments. In practice, if no experimental data are available, the prior stochastic models of uncertainties can be used to perform a sensitivity analysis with respect to its parameters. If a few experimental data are available, these 


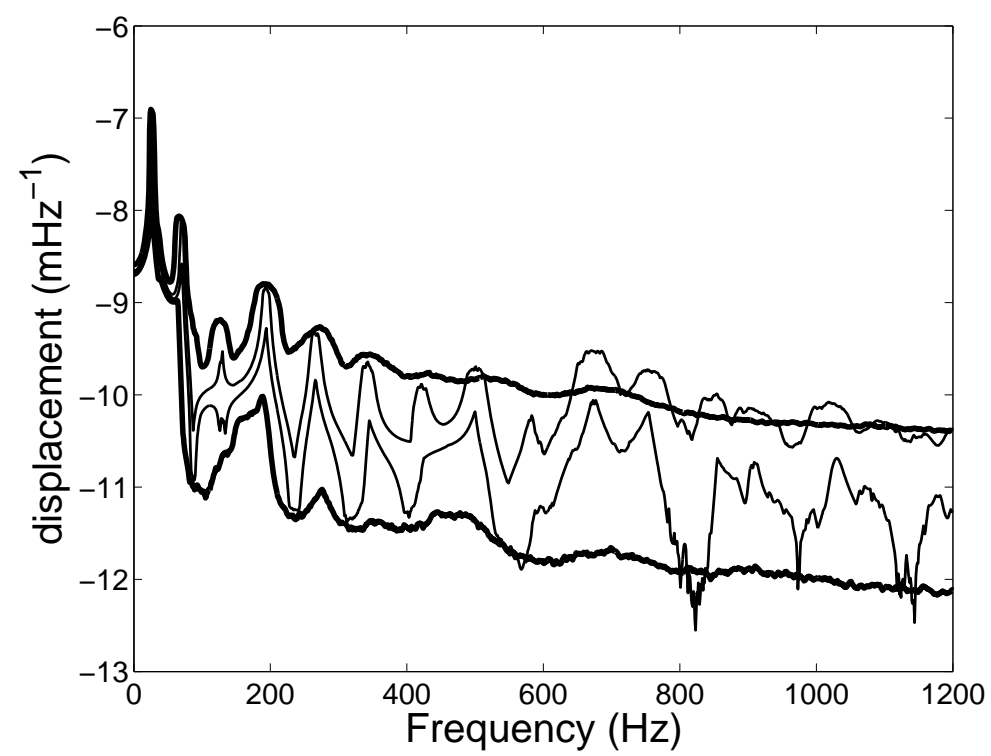

Fig. 15 Random FRF calculated with the optimal stochastic reduced-order computational model: Confidence region (upper and lower thick lines). Experimental FRF: Confidence region (upper and lower thin lines). Observation point $P_{4}$.

parameters can be identified in order to obtain an optimal prior probabilistic model of uncertainties in following the method proposed in the paper. For the particular cases for which a lot of experimental data would be available, a posterior probabilistic model of system-parameters uncertainties could be estimated using the Bayes method (if the stochastic dimension of the uncertain system parameters is not too high) in presence of modeling errors.

Acknowledgements This research was supported by the "Agence Nationale de la Recherche", Contract TYCHE, ANR-2010-BLAN-0904.

\section{A Nonparametric probabilistic approach of uncertainties.}

The nonparametric probabilistic approach of uncertainties of modeling errors has been introduced in [29] (see for instance $[1 ; 7]$ for experimental validations and uncertainty quantification) as a possible way to take into account uncertainties induced by modeling errors. The nonparametric probabilistic approach consists in directly constructing the stochastic modeling of the operators of the nominal computational model and can be used even if no available data are available. In the context of structural dynamics, this approach consists in replacing the matrices of nominal reduced model by random matrices for which the probability distributions are constructed by using the maximum entropy principle with constraints defined by the available information. Therefore, the positive-definite symmetric $(n \times n)$ real matrices $[M],[D]$ and $[K]$ of the reduced mean system are replaced by the random matrices $[\mathbf{M}],[\mathbf{D}]$ and $[\mathbf{K}]$ with values in the set of all positive-definite symmetric $(n \times n)$ real matrices.

Let $A$ denote $M, D$ or $K$. Then, the Cholesky factorization of matrix $[A]$ yields $[A]=\left[L_{A}\right]^{T}\left[L_{A}\right]$. Random matrix $[\mathbf{A}]$ is then constructed such that $[\mathbf{A}]=\left[L_{A}\right]^{T}\left[\mathbf{G}_{A}\right]\left[L_{A}\right]$. Let $E\{$.$\} be the mathematical expectation$ and let $0 \leq \varepsilon_{0} \ll 1$ be a positive number as small as one wants. The random matrix $\left[\mathbf{G}_{A}\right]$ is written as $\left[\mathbf{G}_{A}\right]=\frac{1}{1+\varepsilon_{0}}\left\{\left[\mathbf{G}_{0}\right]+\varepsilon_{0}[I]\right\}$ in which $[I]$ is the $(n \times n)$ identity matrix and where the probability density function of the random matrix $\left[\mathbf{G}_{0}\right]$ is then constructed with the following available information. The random matrix $\left[\mathbf{G}_{0}\right]$ (1) must be positive definite almost surely, (2) has a mean value which is equal to the identity matrix and (3) must verify the inequality $\left|E\left\{\log \left(\operatorname{det}\left[\mathbf{G}_{0}\right]\right)\right\}\right|<+\infty$ in order that the stochastic response of the stochastic computational dynamical model be a second-order random stochastic process. The statistical fluctuations of $\left[\mathbf{G}_{0}\right]$ are controlled by the dispersion parameter $\delta_{A}$ which is defined by

$$
\delta_{A}=\left\{\frac{1}{n} E\left\{\left\|\left[\mathbf{G}_{0}\right]-[I]\right\|\right\}_{F}^{2}\right\}^{1 / 2}
$$


and which must be chosen such that $0<\delta_{A}<\sqrt{(n+1) /(n+5)}$. The probability density function $p_{\left[\mathbf{G}_{0}\right]}([G])$ of the random matrix $\left[\mathbf{G}_{0}\right]$ is then defined by

$$
p_{\left[\mathbf{G}_{0}\right]}([G])=\mathbb{1}_{\mathbb{M}_{n}^{+}(\mathbb{R})}([G]) \times C_{A} \times(\operatorname{det}[G])^{\frac{n+1}{2}\left(\delta_{A}^{-2}-1\right)} \times e^{-\frac{n+1}{2} \delta_{A}^{-2} \operatorname{tr}[G]},
$$

in which the positive constant $C_{A}$ is such that

$$
C_{A}=(2 \pi)^{-\frac{n(n-1)}{4}}\left(\frac{n+1}{2 \delta_{A}^{2}}\right)^{\frac{n(n+1)}{2} \delta_{A}^{-2}}\left\{\Pi_{j=1}^{n} \Gamma\left(\frac{n+1}{2 \delta_{A}^{2}}+\frac{1-j}{2}\right)\right\}^{-1},
$$

where $\Gamma(z)$ is the gamma function defined for $z>0$ by $\Gamma(z)=\int_{0}^{+\infty} t^{z-1} e^{-t} d t$. Note that Eq. (17) shows that $\left\{[\mathbf{G}]_{j k}, 1 \leq j \leq k \leq n\right\}$ are dependent random variables. Concerning the generator of independent realization, the random matrix $\left[\mathbf{G}_{0}\right]$ is written as $\left[\mathbf{G}_{0}\right]=[\mathbf{L}]^{T}[\mathbf{L}]$, in which $[\mathbf{L}]$ is an upper triangular random matrix with values in $\mathrm{M}_{n}(\mathrm{R})$ such that:

(1) the random variables $\left\{[\mathbf{L}]_{j j^{\prime}}, j \leq j^{\prime}\right\}$ are independent;

(2) for $j<j^{\prime}$, real-valued random variables $[\mathbf{L}]_{j j^{\prime}}$ can be written as $[\mathbf{L}]_{j j^{\prime}}=\sigma_{m} U_{j j^{\prime}}$ in which $\sigma_{m}=\delta_{A} / \sqrt{n+1}$ and where $U_{j j^{\prime}}$ is a real-valued Gaussian random variable with zero mean and variance equal to 1 ;

(3) for $j=j^{\prime}$, positive-valued random variables $[\mathbf{L}]_{j j}$ can be written as $[\mathbf{L}]_{j j}=\sigma_{m} \sqrt{2 V_{j}}$ in which $V_{j}$ is a positive-valued gamma random variable whose probability density function $p_{V_{j}}(v)$ with respect to $d v$ is written as

$$
p_{V_{j}}(v)=\mathbb{1}_{\mathbb{R}^{+}}(v) \frac{1}{\Gamma\left(\frac{n+1}{2 \delta_{A}^{2}}+\frac{1-j}{2}\right)} v^{\frac{n+1}{2 \delta_{A}^{2}}-\frac{1+j}{2}} e^{-v},
$$

in which $\delta_{A}$ is the dispersion parameter defined by Eq. (16).

\section{References}

1. Batou A, Soize C, Corus M (2011) Experimental identification of an uncertain computational dynamical model representing a family of structures. Computers and Structures 89(13-14):1440-1448.

2. Beck, J.L., Katafygiotis, L.S. (1998) Updating models and their incertainties. I: Bayesian statistical framework. Journal of Engineering Mechanics 124(4):455-461.

3. Bowman AW, Azzalini A (1997) Applied Smoothing Techniques for Data Analysis. Oxford University Press.

4. Das S., Ghanem R, Spall J (2008) Asymptotic sampling distribution for polynomial chaos representation of data : A maximum-entropy and fisher information approach. SIAM Journal on Scientific Computing 30(5):2207-2234.

5. Debusschere BJ, Najm HN, Pebay PP, Knio OM, Ghanem R, Le Maitre OP (2004) Numerical challenges in the use of polynomial chaos representations for stochastic processes. SIAM Journal on Scientific Computing 26(2):698-719.

6. Desceliers C, Soize C, Ghanem R (2007) Identification of chaos representations of elastic properties of random media using experimental vibration tests. Computational mechanics 39(6):831-838.

7. Durand JF, Soize C, Gagliardini L (2008) Structural-acoustic modeling of automotive vehicles in presence of uncertainties and experimental identification and validation. J. Acoust. Soc. Am. 124(3):1513-1525.

8. Ganapathysubramanian B, Zabaras N (2007) Sparse grid collocation schemes for stochastic natural convection problems. Journal of Computational Physics 225(1):652-685.

9. Ghanem R (1999) Ingredients for a general purpose stochastic finite elements formulation. Comput. Methods Appl. Mech. Eng. 168(1-4) (1999) 19-34.

10. Ghanem R, Spanos PD (2003) Stochastic Finite Elements: A Spectral Approach, revised edition. Dover Publications, New-York.

11. Ghanem R, Doostan R (2006) Characterization of stochastic system parameters from experimental data: A bayesian inference approach. Journal of Computational Physics 217(1):63-81.

12. Ghanem R, Doostan R, Red-HorseJ (2008) A probability construction of model validation. Comput. Methods Appl. Mech. Eng. 197(29-32):2585-2595.

13. Ghosh D, Farhat C (2008) Strain and stress computation in stochastic finite element methods. International Journal for Numerical Methods in Engineering 74(8):1219-1239.

14. Guilleminot J, Soize C, On the statistical dependence for the components of random elasticity tensors exhibiting material symmetry properties. Journal of Elasticity, Accepted for publication, June 16 (2012).

15. Hughes TJR (1987) The Finite Element Method: Linear Static and Dynamic Finite Element Analysis. Prentice Hall, Englewood Cliffs, New Jersey.

16. Jaynes ET (1957) Information theory and statistical mechanics. Physical Review 106(4):620-630 and 108(2):171-190

17. Kaipio J, Somersalo E (2005) Statistical and Computational Inverse Problems. Springer-Verlag, New York. 
18. Kapur JN, Kesavan HK (1992) Entropy Optimization Principles with Applications. Academic Press, San Diego.

19. Le Maitre OP, Knio OM (2010) Spectral Methods for Uncertainty Quantification with Applications to Computational Fluid Dynamics. Springer, Heidelberg.

20. Mace RR, Worden W, Manson G (editors) (2005) Uncertainty in structural dynamics. Special issue of the Journal of Sound and Vibration 288(3):431-790.

21. Matthies HG (2008) Stochastic finite elements: Computational approaches to stochastic partial differential equations. Zamm-Zeitschrift für Angewandte Mathematik und Mechanik 88(11) (2008) 849-873.

22. Matthies HG, Keese A (2005) Galerkin methods for linear and nonlinear elliptic stochastic partial differential equations. Comput. Methods Appl. Mech. Eng. 194(12-16):1295-1331.

23. Najm HN (2009) Uncertainty quantification and polynomial chaos techniques in computational fluid dynamics. Journal Review of Fluid Mechanics 41:35-52.

24. Nouy A (2010) Proper Generalized Decompositions and Separated Representations for the Numerical Solution of High Dimensional Stochastic Problems. Archives of Computational Methods in Engineering 17(4):403434.

25. Schueller GI (Ed.) (1997) A state-of-the-art report on computational stochastic mechanics. Probabilistic Engineering Mechanics 12(4):197-313.

26. Schueller GI (Ed.) (2005) Computational methods in stochastic mechanics and reliability analysis. Comput. Methods Appl. Mech. Eng. 194(12-16):1251-1795.

27. Serfling RJ (1980) Approximation Theorems of Mathematical Statistics. John Wiley \& Sons.

28. Shannon CE (1948) A mathematical theory of communication. Bell System Technology Journal 27:379-423 and 27:623-659.

29. Soize C (2000) A nonparametric model of random uncertainties on reduced matrix model in structural dynamics. Probabilistic Engineering Mechanics 15(3):277-294

30. Soize C, Ghanem R (2004) Physical systems with random uncertainties : Chaos representation with arbitrary probability measure. SIAM Journal on Scientific Computing 26(2):395-410.

31. Soize C (2008) Tensor-valued random fields for meso-scale stochastic model of anisotropic elastic microstructure and probabilistic analysis of representative volume element size. Probabilistic Engineering Mechanics 23(2-3):307-323.

32. Soize C (2010) Generalized probabilistic approach of uncertainties in computational dynamics using random matrices and polynomial chaos decomposition, International Journal for Numerical Methods in Engineering 81(8):939-970.

33. Spall JC (2003) Introduction to Stochastic Search and Optimization. John Wiley and Sons, Hoboken, New Jersey.

34. Spanos PD, Ghanem R (1989) Stochastic finite element expansion for random media. Journal of Engineering Mechanics, ASCE 115(5):1035-1053

35. Ta QA, Clouteau D, Cottereau R (2010) Modeling of random anisotropic elastic media and impact on wave propagation. European Journal of Computational Mechanics 19(1-2-3):241-253.

36. Vanmarcke E, Shinozuka M, Nakagiri S, Schueller GI, Grigoriu M (1986) Random-fields and stochastic Finite-Element. Structural Safety 3(3-4):143-166.

37. Walter E, Pronzato L (1997) Identification of Parametric Models from Experimental Data. Springer, Berlin.

38. Wan XL, Karniadakis GE (2006) Multi-element generalized polynomial chaos for arbitrary probability measures. SIAM Journal on Scientific Computing 28(3):901-928.

39. Xiu DB, Karniadakis GE (2002) Wiener-Askey polynomial chaos for stochastic differential equations. SIAM Journal on Scientific Computing 24(2):619-644.

40. Xu XF (2007) A multiscale stochastic finite element method on elliptic problems involving uncertainties. Comput. Methods Appl. Mech. Eng. 196(25-28):2723-2736. 